\title{
Article \\ Dietary Intake of Milk Thistle Seeds as a Source of Silymarin and Its Influence on the Lipid Parameters in Nonalcoholic Fatty Liver Disease Patients
}

\author{
Aleksandra Kołota * (D) and Dominika Głąbska (D) \\ Department of Dietetics, Institute of Human Nutrition Sciences, \\ Warsaw University of Life Sciences (SGGW-WULS), 159c Nowoursynowska Street, 02-776 Warsaw, Poland; \\ dominika_glabska@sggw.edu.pl \\ * Correspondence: aleksandra_kolota@sggw.edu.pl; Tel.: +48-22-5937186
}

check for

updates

Citation: Kołota, A.; Głąbska, D. Dietary Intake of Milk Thistle Seeds as a Source of Silymarin and Its Influence on the Lipid Parameters in Nonalcoholic Fatty Liver Disease Patients. Appl. Sci. 2021, 11, 5836. https://doi.org/10.3390/ app11135836

Academic Editor: Monica Gallo

Received: 16 May 2021

Accepted: 19 June 2021

Published: 23 June 2021

Publisher's Note: MDPI stays neutral with regard to jurisdictional claims in published maps and institutional affiliations.

Copyright: (c) 2021 by the authors. Licensee MDPI, Basel, Switzerland. This article is an open access article distributed under the terms and conditions of the Creative Commons Attribution (CC BY) license (https:// creativecommons.org/licenses/by/ $4.0 /)$.
Abstract: Nonalcoholic fatty liver disease (NAFLD), which is associated with obesity, is the most common reason for chronic hepatitis in developed countries. Its therapy includes a low-energy diet, physical activity and also various antioxidative compounds, such as milk thistle (Silybum Marianum) seeds, which are a natural source of silymarin. The aim of the study was to assess the influence of 3 months of therapy including dietary and physical activity recommendations combined with a daily milk thistle supply on the biochemical parameters of NAFLD patients. The study was conducted on a group of 20 patients (11 females, 9 males) with NAFLD and excessive body mass diagnosed. For 3 months, participants received daily doses of $7.1 \mathrm{~g}$ of milk thistle as a source of silymarin (210 $\mathrm{mg}$ a day). They also received dietary and physical activity recommendations and were monitored every 2 weeks. Their diet, body composition and biochemical parameters were assessed. It was observed that the studied patients did not follow their dietary recommendations, as when comparing the baseline diet and the diet after 3 months, there were no differences (for the vast majority of nutrients; $p<0.05$ ). Patients also did not follow their physical activity recommendations, as when comparing baseline results with those after 3 months, there were no differences (for the vast majority of parameters; $p<0.05$ ). However, when comparing baseline biochemical parameters and those after 3 months, it was indicated that blood triglyceride levels (140.36 \pm 14.92 vs. $110.04 \pm 37.23 \mathrm{mg} / \mathrm{dL} ; p=0.0017)$ and gamma-glutamyltransferase (GGT) activity decreased $(23.03 \pm 12.87$ vs. $12.77 \pm 5.24 \mathrm{U} / \mathrm{L} ; p=0.0008)$. As the diet and physical activity of the studied patients were not changed within the studied period, it may be supposed that the beneficial decrease in blood triglyceride levels and GGT activity were obtained due to the milk thistle supply as a source of silymarin. It may be indicated that including milk thistle in an everyday diet may be an additional recommendation for NAFLD patients, as it allows one to obtain beneficial effects, even if the other recommendations are not followed.

Keywords: nonalcoholic fatty liver disease; milk thistle; Silybum Marianum; silymarin; obesity; diet; body mass; lipid profile; liver enzymes

\section{Introduction}

Liver diseases are a serious global health problem contributing worldwide to about 2 million deaths per year [1]. In the developed countries, the most common reason for chronic hepatitis is nonalcoholic fatty liver disease (NAFLD), as in various countries its frequency is attributed to $12-32 \%$ [2]. NAFLD is associated with lipid accumulation in the liver, as it is defined as steatosis in over $5 \%$ of hepatocytes observed in individuals neither consuming excessive amounts of alcohol nor diagnosed with concurrent liver diseases [3], and it may develop into nonalcoholic steatohepatitis (NASH), progressing to liver cirrhosis $[4,5]$. As a result, NAFLD is indicated as one of the most important factors 
contributing to gastrointestinal cancers, including especially hepatocellular carcinoma, which is the most common type of liver cancer [6].

The pathogenesis of NAFLD is complex, associated with various genetic, metabolic and environmental factors [7], resulting from oxidative stress, lipid peroxidation, mitochondrial disfunction and release of pro-inflammatory cytokines [4]. NAFLD is commonly concurrent with other diseases and disorders, including obesity, diabetes, dyslipidemia, hypertension, cardiovascular diseases and chronic renal insufficiency [8-10]. Taking into consideration that the majority of indicated conditions are included as elements of metabolic syndrome, it is suggested that NAFLD may be a liver manifestation of a metabolic syndrome [11].

It seems that a crucial element for NAFLD development is excessive body mass, as about $80 \%$ of NAFLD patients are obese [12], which corresponds with the increasing prevalence of obesity and NAFLD observed worldwide at the same time [2]. It may be indicated that both diseases result from following the western diet, associated with a high intake of simple carbohydrates and fat, leading to lipid accumulation and contributing to the development of liver steatosis [13]. Taking this into account, the major element of the behavioral therapy of NAFLD is associated with lifestyle changes in order to reduce body mass, which must be accompanied by the control of metabolic syndrome risk factors or its treatment $[14,15]$. It is recommended to combine dietary modifications (low-energy diet) and physical activity, as such a combination is the most effective form of therapy for NAFLD and obesity $[16,17]$.

In spite of the fact that there are no uniform standards of NAFLD treatment due to its complex pathophysiology, various medications were found to reduce liver steatosis, fibrosis and inflammation [18]. Recently, growing attention is paid to the possibility of using natural therapeutic options and herbal treatment in NAFLD therapy, not only due to their effectiveness and low risk of side effects but also due to their availability for patients [19]. The most promising options are grapes, being a source of resveratrol; milk thistle (Silybum Marianum) seeds, being a source of silymarin; or more complex products, such as ginkgo, ginseng, turmeric, garlic, ginger or cinnamon [20]. However, in a number of studies on NAFLD, not natural products but rather their extracts were studied while passed in a form of dietary supplements [21,22]; this is in spite of the fact that the natural product may also be an effective therapeutic option provided in an everyday diet as a source of various beneficial compounds. Moreover, for patients with metabolic syndrome, as NAFLD individuals commonly are [10,23], with a specific pharmacotherapy associated with their condition [24], using various dietary supplements may be potentially harmful due to the high risk of excessive supply of active components; thus, it may be especially beneficial for them to use natural products instead of supplements.

Milk thistle is a well-known plant that was used for many years for the treatment of the liver and gallbladder due to its content of natural polyphenols and flavonolignans, such as isosilychristin, silychristins A and B, silydianin and silybins A and B, as well as isosilybins A and B [25]; this complex is called silymarin, presenting antioxidative and antifibrotic activities [26,27]. Such effects may be of great value for NAFLD patients, as oxidative processes play an important role in pathogenesis and progression of this disease [4]. The studies conducted to date in animal models indicated positive effects of milk thistle extract reducing hepatic steatosis [28]. Moreover, it is supposed that silymarin may reduce oxidative stress and resultant cytotoxicity, protecting hepatocytes from damage [29]. Silymarin acts as a free radical scavenger, and it modulates the action of enzymes associated with cell damage, hepatic fibrosis and cirrhosis, as was observed for patients with NAFLD, including those with cirrhosis [29]. At the same time, it was stated that in NAFLD patients, silymarin reduces inflammatory parameters, liver enzyme blood levels and hepatic steatosis [30]. Moreover, it is indicated that silymarin may reduce the development of atherosclerosis, also being important for individuals with NAFLD [31]. As a result, for NAFLD patients, combining a low-energy diet, physical activity and milk thistle supply may exert a positive 
influence on liver function [32]. It should be possible, as milk thistle is often well tolerated by patients and any side effects are very rare [29].

Taking this into account, the aim of the current study was to assess the influence of 3 months of therapy including dietary and physical activity recommendations combined with a daily milk thistle supply on the biochemical parameters of NAFLD patients.

\section{Materials and Methods}

\subsection{Study Design}

The study was conducted based on the guidelines of the Declaration of Helsinki. It was approved by the Ethics Committee of the Central Clinical Hospital of the Ministry of Interior in Warsaw (9/2016). Written informed consent was provided by all participants of the study.

The study was based on a monitored intervention, including dietary and physical activity recommendations combined with daily milk thistle supply. The milk thistle was provided to participants, and each participant was supervised by a physician and dietitian to monitor their wellbeing as well as to control following recommendations. Participants were not excluded from the study if they did not follow the dietary and physical activity recommendations, but only if they did not consume the provided daily milk thistle doses.

\subsection{Participants}

The study was conducted on a group of patients with NAFLD diagnosed by a gastroenterologist based on an ultrasound examination (USG) with liver steatosis observed, which was confirmed to not be associated with alcohol overconsumption-alcoholic fatty liver disease (AFLD) was ruled out.

The inclusion criteria were as follows:

- $\quad$ Patients aged $>18$ years;

- NAFLD diagnosed and confirmed by gastroenterologist;

- Liver steatosis diagnosed and confirmed by gastroenterologist;

- Daily alcohol intake lower than $20 \mathrm{~g}$ - assessed based on the dietary interview;

- Excessive body mass—assessed based on body mass index (BMI), for BMI $\geq 25 \mathrm{~kg} / \mathrm{m}^{2}$.

- The exclusion criteria were as follows:

- Pregnancy or lactation;

- Oncological treatment;

- Diseases potentially responsible for liver steatosis, including hemochromatosis, viral hepatitis B and C, autoimmune hepatitis and Wilson's disease;

- Other factors potentially responsible for liver steatosis, including medications and toxins.

Participants of the study were recruited and qualified for the study by gastroenterologists, and they were informed about the study either by them or based on the local press advertisement.

In total, 21 patients were recruited for the study, but within the study, 1 of them did not consume the provided daily Silybum Marianum doses, resulting in exclusion from the study. The final sample size analyzed within the study was 20 participants, including 11 female participants and 9 male participants. The general baseline characteristics of the studied group of participants are presented in Table 1.

Table 1. The general baseline characteristics of the studied group of participants.

\begin{tabular}{cccccc}
\hline \multicolumn{2}{c}{ Parameter } & Total $(\boldsymbol{n = 2 0 )}$ & Females $(\boldsymbol{n = 1 1 )}$ & Males $(\boldsymbol{n = 9 )}$ & $\boldsymbol{p}^{* *}$ \\
\hline \multirow{2}{*}{ Age (years) } & Mean \pm SD & $52.3 \pm 10.9$ & $56.1 \pm 11.9$ & $47.6 \pm 7.9$ & 0.0869 \\
& Median (min-max) & $51.0(36-76)$ & $55.0(36-76)$ & $47.0(38-64)$ & $33.2 \pm 3.9$ \\
BMI $\left(\mathrm{kg} / \mathrm{m}^{2}\right)$ & Mean \pm SD & $36.5 \pm 5.9$ & $39.3 \pm 6.1$ & 0.0873 \\
& Median (min-max) & $36.3(26.6-55.7)$ & $38.8(32.8-55.7) *$ & $34.2(26.6-37.0)$ \\
\hline
\end{tabular}

${ }^{*}$ nonparametric distribution (Shapiro-Wilk test; $\left.p \leq 0.05\right)$; ${ }^{* *}$ compared while using t-Student test or Mann-Whitney test (based on distribution). 


\subsection{Experimental Procedures}

Participants received milk thistle seeds (HerbaNordPol-Gdańsk LLC, Nowy Staw, Poland) as a source of silymarin. Taking this into account, the daily dose of milk thistle was calculated based on the required silymarin dose. In clinical studies, various silymarin doses are applied, differing from $210 \mathrm{mg}$ to $1200 \mathrm{mg}$ a day [33]. At the same time, silymarin is indicated to be well tolerated, without any side effects, when applied in doses from $200 \mathrm{mg}$ to $900 \mathrm{mg}$, but doses higher than $1500 \mathrm{mg}$ may cause excessive bile production and laxative effects [34]. The silymarin content in the applied grinded milk thistle seeds was assessed based on the method of high-performance liquid chromatography (HPLC) [25] in a certified laboratory of the Institute of Natural Fibres and Medicinal Plants in Poznan (Poland), and it was observed to amount to $2.96 \%$, including silybins A and B (1.76\%), silychristins $(0.78 \%)$, isosilybins A and B $(0.40 \%)$ and silydianin $(0.06 \%)$. On the basis of this assessment, participants received a daily dose of $7.1 \mathrm{~g}$ of milk thistle $(210 \mathrm{mg}$ of silymarin). They received it for 3 months, and they were provided daily servings in air-tight containers. They received them during their medical and dietary checkup, every 2 weeks for the following 14 days.

Participants received commercially available grinded milk thistle seeds and were instructed to consume them within their regular dishes, as the product is characterized by a neutral taste, smell and flavor; thus, it may be added to any product. They were informed that it may be added, e.g., to yoghurt, soup, salad, etc.

At the same time, participants received dietary and physical activity recommendations as essential elements of the therapy for NAFLD and obesity [16,17]. Their diet, physical activity and milk thistle consumption were monitored every 2 weeks, and not following the dietary and physical activity recommendations they were encouraged to follow was not a reason to exclude participants from the study.

The dietary and physical activity recommendations were individually developed for each participant based on their dietary and physical activity baseline assessments, as well as their energy requirement that was calculated based on the Harris-Benedict equation [35]. They were recommended to follow a diet with a $600 \mathrm{kcal}$ energy deficit per day, which is in agreement with recommendations for NAFLD patients to meet the energy deficit of $500 \mathrm{kcal}-750 \mathrm{kcal}$ within their diet [36]. Based on the 3-day dietary record, provided by each participant, the usual intake was established and individual dietary recommendations were planned. The recommendations included the energy deficit, an adequate number of meals per day (4-5), intervals between meals of about $3 \mathrm{~h}$, changes of food product choice (increased intake of whole grain cereals, vegetables, no sweets and fast foods) and recommended methods of thermal treatment (cooking or baking instead of frying), which is in agreement with general recommendations for NAFLD patients and within the Mediterranean dietary pattern [37]. The diet that was planned for each participant was individualized, taking into account concurrent diseases. Each patient received individual recommendations as well as individual menus with information on how to use them and how to diversify them.

At the same time, physical activity recommendations were developed to obtain an additional deficit of $400 \mathrm{kcal}$, which is also in agreement with recommendations for NAFLD patients [36]. The physical activity recommendations were associated with moderate intensity physical activity as recommended for NAFLD patients [38], and the recommended activity was based on a target daily number of steps, as well as encouraging patients to walk, jog, cycle and swim, if possible [36]. Patients were recommended to engage in physical activity for at least 30-60 min and at least 3-5 days a week, and the detailed recommendations were based on guidelines for NAFLD patients [39].

\subsection{Measurements}

\subsubsection{Physiological Parameters}

Body mass was assessed using a calibrated weighing scale, with an accuracy of $\pm 0.1 \mathrm{~kg}$, and height was measured using a stadiometer, with an accuracy of $\pm 0.5 \mathrm{~cm}$. The waist 
and hip circumferences were assessed using a non-elastic flexible measuring tape, with an accuracy of $\pm 0.5 \mathrm{~cm}$. All the measurements were conducted by a qualified dietitian. Based on the conducted measurements, BMI [40] and waist-to-hip ratio (WHR) [41] were obtained.

Body composition was assessed using a bioelectrical impedance measurement conducted with a frequency of $50 \mathrm{kHz}$ by a BIA 101/SC device (Akern Srl, Firenze, Italy). The measurement was conducted by a qualified dietitian using a commonly applied methodology and procedure - after a day characterized by a typical diet, without any alcohol, coffee or other caffeine beverages and moderate physical activity, in a fasting state [42].

Diet was assessed using the method of a 3-day dietary record as recommended by the Food and Agriculture Organization of the United Nations (FAO) [43]. The serving sizes were allowed to be either weighted or recorded in household measures to provide accurate assessment of the intake. Based on the recorded intake, the energy value of the diet and nutrient intake were calculated using a Polish table of the nutritional value of foods [44] and Polish software-Energia 4.1 (1997/2006 by Andrzej Miegoć, Warsaw, Poland). The daily intake was compared with the Polish recommendations on the estimated average requirement (EAR) or adequate intake (AI) level, depending on the nutrient [45].

As an additional element of dietary assessment, the diet quality indices were calculated. The atherogenic index of Keys was calculated based on the intake of saturated fatty acids (SFA), polyunsaturated fatty acids (PUFA) and cholesterol, according to the following formula: the atherogenic index of Keys $=1.35 \times(2 \times \%$ SFA $-\%$ PUFA $)+1.5$ $\times \sqrt{ }($ Cholesterol/1000 kcal), while: \%SFA and \%PUFA present $\%$ of energy value from indicated fatty acids, and cholesterol expressed in mg [46].

Physical activity was monitored using a SenseWear Armband Pro3 (SenseWear, BodyMedia, Pittsburgh, PA, USA), which is a monitor of energy expenditure, physical activity level and number of steps, as well as time spent while moving. The measurement was conducted for 3 consecutive days while the monitor was placed on the upper dominant arm.

\subsubsection{Biochemical Parameters}

The biochemical parameters were assessed based on the blood samples collected in a fasting state and centrifuged at $3500 \times \mathrm{g}$ rpm at $4{ }^{\circ} \mathrm{C}$ for $15 \mathrm{~min}$. The obtained blood plasma samples were stored at $-21^{\circ} \mathrm{C}$ until analysis.

The triglyceride level in plasma was measured by a spectrophotometric method, while the Liquick Cor-TG (PZ Cormay, Łomianki, Poland) was used, and the results are expressed as $\mathrm{mg} / \mathrm{dL}$.

The total cholesterol level in plasma was measured by a spectrophotometric method, while the Liquick Cor-CHOL (PZ Cormay, Łomianki, Poland) was used, and the results are expressed as $\mathrm{mg} / \mathrm{dL}$.

The high-density lipoprotein (HDL) cholesterol level in plasma was measured by a spectrophotometric method, while the Cormay HDL Direct (PZ Cormay, Łomianki, Poland) was used, and the results are expressed as $\mathrm{mg} / \mathrm{dL}$.

The low-density lipoprotein (LDL) cholesterol level in plasma was measured by a spectrophotometric method, while the Cormay LDL Direct (PZ Cormay, Łomianki, Poland) was used, and the results are expressed as $\mathrm{mg} / \mathrm{dL}$.

The alanine aminotransferase (ALT) activity in plasma was measured by a spectrophotometric method, while the Liquick Cor-ALAT (PZ Cormay, Łomianki, Poland) was used, and the results are expressed as $\mathrm{U} / \mathrm{L}$.

The gamma-glutamyltransferase (GGT) activity in plasma was measured by a spectrophotometric method, while the Liquick Cor-GGT (PZ Cormay, Łomianki, Poland) was used, and the results are expressed as $\mathrm{U} / \mathrm{L}$.

\subsection{Statistical Analysis}

The distribution was verified while using the Shapiro-Wilk test. Afterward, the results were compared using the t-Student test or Mann-Whitney test (based on distribution), as well as the $\mathrm{chi}^{2}$ test. 
During analysis, participants following and not following the dietary and physical activity recommendations were compared. To conduct this comparison, their following of the recommendations was assessed. The following of dietary recommendations was assessed based on the energy deficit obtained after 3 months-as the deficit of $600 \mathrm{kcal}$ was recommended, participants were divided into those following recommendations (of deficit $>500 \mathrm{kcal}$ ) and those not following recommendations (of deficit $<500 \mathrm{kcal}$ ). The following of physical activity recommendations was assessed based on the physical activity duration obtained after 3 months - as the physical activity duration of at least 30-60 min was recommended, participants were divided into those following recommendations (of physical activity duration $>30 \mathrm{~min}$ ) and those not following recommendations (of physical activity duration $<30 \mathrm{~min}$ ).

The values of $p \leq 0.05$ were interpreted as statistically significant differences. The statistical analysis was conducted using the Statistica 13.1. software (StatSoft Inc., Tulsa, OK, USA) and Statgraphics Plus for Windows 5.1 (Statgraphics Technologies Inc., The Plains, VA, USA).

\section{Results}

The body mass and body composition of the female participants at baseline and after 3 months of intervention are presented in Table 2. It was observed that all the parameters were comparable before and after intervention, and no statistically significant differences were observed for female participants $(p>0.05)$. The additional analyses of the body mass and body composition of the participants after 3 months of intervention compared between sub-groups following recommendations and not following recommendations are presented in the Supplementary Materials (Table S1), and they confirmed some effects of following physical activity recommendations. Similarly, analyses of the body mass and body composition of the female participants not following dietary recommendations and following dietary recommendations, respectively, at baseline and after 3 months of intervention, are presented in the Supplementary Materials (Tables S2 and S3, respectively), and they are in agreement with the results for the total studied group.

The body mass and body composition of the male participants at baseline and after 3 months of intervention are presented in Table 3. It was observed that all the parameters were comparable before and after intervention, and no statistically significant differences were observed for male participants $(p>0.05)$. The additional analyses of the body mass and body composition of the male participants not following dietary recommendations and following dietary recommendations, respectively, at baseline and after 3 months of intervention, are presented in the Supplementary Materials (Tables S4 and S5, respectively), and they are in agreement with the results for the total studied group.

Table 2. The body mass and body composition of the female participants at baseline and after 3 months of intervention.

\begin{tabular}{|c|c|c|c|c|c|}
\hline \multirow{2}{*}{ Parameter } & \multicolumn{2}{|c|}{ Baseline } & \multicolumn{2}{|c|}{ After 3 Months of Intervention } & \multirow{2}{*}{$p^{* *}$} \\
\hline & Mean \pm SD & Median (Min-Max) & Mean \pm SD & Median (Min-Max) & \\
\hline Body mass (kg) & $99.2 \pm 18.6$ & $91.2(83.0-142.6) *$ & $96.1 \pm 18.3$ & $89.1(78.2-138.8)$ * & 0.5545 \\
\hline BMI $\left(\mathrm{kg} / \mathrm{m}^{2}\right)$ & $39.3 \pm 6.1$ & $38.8(32.8-55.7)$ & $38.1 \pm 6.4$ & $37.6(29.8-54.2)$ & 0.5253 \\
\hline Waist circumference $(\mathrm{cm})$ & $112.4 \pm 7.5$ & $110.0(104-128)$ & $109.0 \pm 8.9$ & $107.0(96-124)$ & 0.9536 \\
\hline Hip circumference $(\mathrm{cm})$ & $124.6 \pm 10.0$ & $123.0(105-145)$ & $120.9 \pm 8.9$ & $122.0(105-141)$ & 0.3767 \\
\hline WHR & $0.90 \pm 0.04$ & $0.89(0.85-1.01)$ * & $0.90 \pm 0.05$ & $0.88(0.84-0.99)$ * & 0.8696 \\
\hline Fat mass (kg) & $46.2 \pm 11.1$ & $40.8(36.2-73.0) *$ & $52.1 \pm 8.3$ & $49.6(43.1-69.2) *$ & 0.1890 \\
\hline Fat free mass (kg) & $52.9 \pm 8.9$ & $49.4(43.3-69.6)$ & $52.1 \pm 8.4$ & $49.6(43.1-69.2) *$ & 1.0000 \\
\hline Body cell mass (kg) & $25.2 \pm 4.9$ & $25.9(18.7-36.7)$ & $25.1 \pm 3.4$ & $24.4(19.6-32.5)$ & 0.9447 \\
\hline Total body water (L) & $39.9 \pm 6.2$ & $39.0(32.6-51.0)$ & $39.3 \pm 5.7$ & $37.7(32.6-50.6)$ & 0.8137 \\
\hline Extracellular water (L) & $19.7 \pm 3.9$ & $19.5(14.2-25.8)$ & $19.4 \pm 3.6$ & $19.4(14.2-26.5)$ & 0.8538 \\
\hline Intracellular water (L) & $20.2 \pm 3.3$ & $19.9(15.2-27.1)$ & $19.9 \pm 2.7$ & $19.5(15.1-25.2)$ * & 0.6695 \\
\hline
\end{tabular}

BMI—body mass index; WHR—waist-to-hip ratio; * nonparametric distribution (Shapiro-Wilk test; $p \leq 0.05$ ); ${ }^{* *}$ compared while using $\mathrm{t}$-Student test or Mann-Whitney test (based on distribution). 
Table 3. The body mass and body composition of the male participants at baseline and after 3 months of intervention.

\begin{tabular}{|c|c|c|c|c|c|}
\hline \multirow{2}{*}{ Parameter } & \multicolumn{2}{|c|}{ Baseline } & \multicolumn{2}{|c|}{ After 3 Months of Intervention } & \multirow{2}{*}{$p^{* *}$} \\
\hline & Mean \pm SD & Median (Min-Max) & Mean \pm SD & Median (Min-Max) & \\
\hline Body mass (kg) & $103.4 \pm 14.2$ & $104.6(77.0-129.4)^{*}$ & $98.7 \pm 14.9$ & $100.4(72.7-125.3) *$ & 0.5007 \\
\hline $\operatorname{BMI}\left(\mathrm{kg} / \mathrm{m}^{2}\right)$ & $33.2 \pm 3.9$ & $34.2(26.6-37.0)$ & $31.6 \pm 3.9$ & $31.8(25.2-36.3)$ & 0.4190 \\
\hline Waist circumference $(\mathrm{cm})$ & $113.4 \pm 9.8$ & $113.0(94.0-133.0)$ & $107.7 \pm 11.8$ & $103.0(92.0-132.0)$ & 0.3109 \\
\hline Hip circumference $(\mathrm{cm})$ & $112.4 \pm 6.0$ & $113.0(97.0-133.0)$ & $108.9 \pm 7.9$ & $109.0(92.0-117.0)$ & 0.3005 \\
\hline WHR & $1.90 \pm 0.05$ & $0.99(0.94-1.12)^{*}$ & $0.98 \pm 0.05$ & $0.88(0.92-1.12)^{*}$ & 0.9271 \\
\hline Fat mass (kg) & $35.6 \pm 10.3$ & $36.6(20.5-53.2)$ & $30.9 \pm 9.7$ & $29.9(19.1-48.8)$ & 0.3372 \\
\hline Fat free mass (kg) & $67.9 \pm 7.1$ & $68.3(54.7-76.2)$ & $67.8 \pm 7.4$ & $68.5(52.4-76.5)$ & 0.9743 \\
\hline Body cell mass (kg) & $30.1 \pm 5.9$ & $30.3(23.6-42.3)$ & $28.9 \pm 2.5$ & $29.2(25.5-33.4)$ & 0.5895 \\
\hline Total body water (L) & $50.1 \pm 4.8$ & $50.0(40.0-55.7)$ & $50.1 \pm 5.4$ & $52.1(38.4-56.0)$ & 0.9891 \\
\hline Extracellular water (L) & $22.1 \pm 2.9$ & $22.1(17.2-25.6)$ & $22.8 \pm 3.0$ & $23.4(16.4-25.8)$ & 0.6165 \\
\hline Intracellular water (L) & $28.0 \pm 3.2$ & $28.9(21.6-31.5)$ & $27.3 \pm 2.4$ & $27.7(22.0-30.6)^{*}$ & 0.5839 \\
\hline
\end{tabular}

BMI—body mass index; WHR—waist-to-hip ratio; * nonparametric distribution (Shapiro-Wilk test; $p \leq 0.05$ ); ${ }^{* *}$ compared while using $\mathrm{t}$-Student test or Mann-Whitney test (based on distribution).

The energy and nutritional value of the diet of the female participants at baseline and after 3 months of intervention are presented in Table 4 . It was observed that all the parameters were comparable before and after intervention, and no statistically significant differences were observed for female participants $(p>0.05)$. The additional analyses of the energy and nutritional value of the diet of the participants after 3 months of intervention compared between sub-groups following recommendations and not following dietary and physical activity recommendations, respectively, are presented in the Supplementary Materials (Tables S6 and S7, respectively), and they indicated some effects of following dietary recommendations for the energy value of the diet and protein and sodium intake. Similarly, analyses of the energy and nutritional value of the diet of the female participants not following dietary recommendations and following dietary recommendations, respectively, at baseline and after 3 months of intervention, are presented in the Supplementary Materials (Tables S8 and S9, respectively), and they are in agreement with the results for the total studied group.

The energy and nutritional value of the diet of the male participants at baseline and after 3 months of intervention are presented in Table 5. It was observed that the vast majority of the parameters were comparable before and after intervention, and no statistically significant differences were observed for male participants $(p>0.05)$. Only the protein (16.44 \pm 1.88 vs. $18.70 \pm 2.52 \%$ of energy; $p=0.0464)$ and vitamin $C$ intake increased ( $56.09 \pm 25.69$ vs. $85.50 \pm 29.35 \mathrm{mg} ; p=0.0326)$. The additional analyses of the energy and nutritional value of the diet of the male participants not following dietary recommendations and following dietary recommendations, respectively, at baseline and after 3 months of intervention, are presented in the Supplementary Materials (Tables S10 and S11, respectively), and they indicated some differences for protein intake.

The nutritional value of the diet compared with the recommended values depending on the age of the female participants at baseline and after 3 months of intervention is presented in Table 6. It was observed that all the parameters were comparable before and after intervention, and no statistically significant differences were observed for female participants $(p>0.05)$. The majority of participating women consumed excessive amounts of protein, sodium, phosphorus, magnesium, iron, zinc and copper, as well as vitamins A, E, C, B1, B2, B6 and B12 and niacin. They also consumed insufficient amounts of carbohydrates, fiber, calcium, iodine, vitamin D and folate. 
Table 4. The energy and nutritional value of the diet of the female participants at baseline and after 3 months of intervention.

\begin{tabular}{|c|c|c|c|c|c|}
\hline \multirow{2}{*}{ Parameter } & \multicolumn{2}{|c|}{ Baseline } & \multicolumn{2}{|c|}{ After 3 Months of Intervention } & \multirow{2}{*}{$p^{* *}$} \\
\hline & Mean \pm SD & Median (Min-Max) & Mean \pm SD & Median (Min-Max) & \\
\hline Energy (kcal) & $1655.8 \pm 416.8$ & $1661.7(789.6-2319.4)$ & $1471.6 \pm 287.6$ & $1422(1137.4-2167.2)$ & 0.2418 \\
\hline Protein (\% of energy) & $20.11 \pm 3.78$ & $19.24(13.81-26.90)$ & $20.75 \pm 3.78$ & $20.82(15.06-27.36)$ & 0.6982 \\
\hline Carbohydrate (\% of energy) & $44.18 \pm 7.15$ & $43.02(32.81-55.59)$ & $42.26 \pm 8.48$ & $41.87(26.82-53.01)$ & 0.5720 \\
\hline Fat (\% of energy) & $35.66 \pm 7.09$ & $35.28(22.02-37.89)$ & $35.74 \pm 8.35$ & $34.41(21.03-48.09)$ & 0.9807 \\
\hline Saturated fat (\% of energy) & $12.93 \pm 2.15$ & $13.51(8.74-15.18)$ & $12.25 \pm 2.29$ & $12.84(8.39-14.93)$ & 0.4823 \\
\hline Sugars ( $\%$ of energy) & $10.37 \pm 5.05$ & $10.81(1.71-18.59)$ & $7.72 \pm 4.44$ & $5.69(3.63-17.82)$ * & 0.1891 \\
\hline Cholesterol (mg) & $310.4 \pm 94.3$ & 316.9 (194.5-438.3) & $268.8 \pm 105.2$ & $244.9(143.9-423.6)$ & 0.3398 \\
\hline Fiber $(\mathrm{g})$ & $20.83 \pm 7.10$ & $21.99(9.04-32.3)$ & $22.64 \pm 8.42$ & $21.38(9.98-37.45)$ & 0.5926 \\
\hline Sodium (mg) & $1863.9 \pm 698.9$ & $1667.9(1264.8-3421.4)$ * & $1616.9 \pm 616.6$ & $1648.9(851.6-2715.1)$ & 0.5909 \\
\hline Potassium (mg) & $3226.0 \pm 910.3$ & $3654.4(1491.9-4322.4)$ & $3526.7 \pm 892.8$ & $3303.8(1832.2-4545.8)$ & 0.4433 \\
\hline Phosphorus (mg) & $1294.3 \pm 320.4$ & $1251.5(663.1-1749.7)$ & $1250.7 \pm 284.3$ & $1245.9(747.3-1796.6)$ & 0.7393 \\
\hline Calcium (mg) & $691.3 \pm 273.2$ & 633.5 (240.6-1079.7) & $619.4 \pm 229.1$ & $646.8(219.6-961.4)$ & 0.5111 \\
\hline Magnesium (mg) & $306.2 \pm 89.5$ & $307.5(130.5-425.5)$ & $320.9 \pm 93.5$ & $309.2(136.3-498.4)$ & 0.7099 \\
\hline Iron (mg) & $11.12 \pm 2.53$ & $11.11(7.00-15.64)$ & $11.57 \pm 2.41$ & $11.08(6.46-14.87)$ & 0.6714 \\
\hline Zinc (mg) & $10.24 \pm 2.84$ & $10.52(5.41-15.29)$ & $10.29 \pm 2.47$ & $9.92(5.78-14.73)$ & 0.9595 \\
\hline Cooper (mg) & $1.31 \pm 0.37$ & $1.42(0.57-1.79)$ & $1.34 \pm 0.36$ & $1.32(0.60-1.96)$ & 0.8286 \\
\hline Manganese (mg) & $5.39 \pm 2.41$ & $5.04(1.10-10.99)$ & $4.85 \pm 2.19$ & $4.90(1.36-9.87)$ & 0.5846 \\
\hline Iodine $(\mu \mathrm{g})$ & $32.56 \pm 12.79$ & $29.20(11.30-57.21)$ & $48.80 \pm 23.86$ & 50.05 (9.64-90.05) & 0.0605 \\
\hline Vitamin A $(\mu \mathrm{g})$ & $879.8 \pm 343.3$ & $762.3(433.6-1471.5)$ & $981.5 \pm 338.4$ & 965.7 (611.0-1579.8) & 0.4881 \\
\hline Vitamin D ( $\mu \mathrm{g})$ & $4.59 \pm 5.57$ & $1.96(0.93-19.05)^{*}$ & $2.92 \pm 2.19$ & $2.25(0.63-6.92)$ & 0.7911 \\
\hline Vitamin E (mg) & $9.33 \pm 2.79$ & $10.37(2.50-12.24) *$ & $10.79 \pm 2.21$ & $10.70(7.81-14.87)$ & 0.8598 \\
\hline Vitamin C (mg) & $85.93 \pm 56.82$ & $86.92(13.52-223.9)$ * & $87.96 \pm 39.99$ & $109.6(24.33-144.07)$ & 0.9238 \\
\hline Vitamin B1 (mg) & $1.02 \pm 0.29$ & $1.00(0.43-1.49)$ & $0.98 \pm 0.21$ & $1.02(0.55-1.28)$ & 0.6799 \\
\hline Vitamin B2 (mg) & $1.70 \pm 0.41$ & $1.82(0.98-2.43)$ & $1.62 \pm 0.35$ & $1.61(1.07-2.01)$ & 0.6292 \\
\hline Vitamin B6 (mg) & $1.64 \pm 0.52$ & $1.66(1.04-2.68)$ & $1.72 \pm 0.46$ & $1.67(0.97-2.51)$ & 0.7204 \\
\hline Vitamin B12 $(\mu \mathrm{g})$ & $4.68 \pm 3.71$ & $3.24(1.75-14.62)$ * & $3.52 \pm 1.31$ & $3.19(2.29-6.52) *$ & 0.1853 \\
\hline Folate $(\mu \mathrm{g})$ & $224.3 \pm 81.91$ & $223.8(67.26-360.7)$ & $253.8 \pm 108.7$ & $233.2(127.7-508.6)$ & 0.4797 \\
\hline Niacin (mg) & $17.40 \pm 3.51$ & $17.53(12.09-22.61)$ & $19.87 \pm 5.93$ & $18.43(10.81-29.36)$ & 0.2481 \\
\hline
\end{tabular}

* nonparametric distribution (Shapiro-Wilk test; $p \leq 0.05$ ); ${ }^{* *}$ compared while using t-Student test or Mann-Whitney test (based on distribution).

Table 5. The energy and nutritional value of the diet of the male participants at baseline and after 3 months of intervention.

\begin{tabular}{|c|c|c|c|c|c|}
\hline \multirow{2}{*}{ Parameter } & \multicolumn{2}{|c|}{ Baseline } & \multicolumn{2}{|c|}{ After 3 Months of Intervention } & \multirow{2}{*}{$p^{* *}$} \\
\hline & Mean \pm SD & Median (Min-Max) & Mean \pm SD & Median (Min-Max) & \\
\hline Energy (kcal) & $2416.0 \pm 592.6$ & $2359.5(1712.8-3591.5)$ & $1885.0 \pm 495.6$ & $1874.0(1416.8-2952.6)$ & 0.0558 \\
\hline Protein (\% of energy) & $16.44 \pm 1.88$ & $15.81(14.62-19.09)$ & $18.70 \pm 2.52$ & $19.45(13.91-21.68)$ & 0.0464 \\
\hline Carbohydrate (\% of energy) & $46.01 \pm 6.41$ & $48.71(37.92-55.58)$ & $43.53 \pm 5.99$ & $42.69(36.76-54.57)$ & 0.4094 \\
\hline Fat (\% of energy) & $36.61 \pm 5.73$ & $34.57(29.77-45.79)$ & $36.47 \pm 5.64$ & $39.09(23.76-41.58)$ & 0.6588 \\
\hline Saturated fat (\% of energy) & $13.45 \pm 3.22$ & $12.91(9.78-20.14)$ & $12.92 \pm 3.69$ & $14.77(6.45-16.43)$ & 0.7528 \\
\hline Sugars (\% of energy) & $10.47 \pm 5.15$ & $12.60(3.82-18.79)$ & $8.51 \pm 4.55$ & $6.53(4.39-18.58)$ * & 0.4026 \\
\hline Cholesterol (mg) & $421.2 \pm 216.8$ & $419.3(114.0-796.1 .3)$ & $331.1 \pm 147.9$ & $332.2(116.1-628.8)$ & 0.3183 \\
\hline Fiber $(\mathrm{g})$ & $20.57 \pm 7.48$ & $19.92(10.48-34.4)$ & $22.26 \pm 4.68$ & $21.82(15.71-29.01)$ & 0.5743 \\
\hline Sodium (mg) & $2807.3 \pm 1124.6$ & $2578.9(1562.6-4956.9)$ & $1971.6 \pm 840.6$ & $1814.8(996.8-3929.7)$ & 0.0931 \\
\hline Potassium (mg) & $3547.1 \pm 869.9$ & $3866.2(2253.2-4654.6)$ & $3531.9 \pm 511.4$ & $3573.5(2732.9-4297.1)$ & 0.9646 \\
\hline Phosphorus (mg) & $1465.7 \pm 332.8$ & $1434.7(976.3-2029.3)$ & $1439.5 \pm 314.6$ & 1566.5 (874.4-1898.6) & 0.8659 \\
\hline Calcium (mg) & $623.1 \pm 304.8$ & $518.5(444.0-1407.2)$ * & $631.2 \pm 129.4$ & $649.9(386.0-817.5)$ & 0.1577 \\
\hline Magnesium (mg) & $318.9 \pm 74.3$ & 339.6 (196.3-441.2) & $346.7 \pm 85.2$ & 341.5 (218.5-462.7) & 0.4714 \\
\hline Iron (mg) & $13.27 \pm 3.32$ & $11.86(9.22-18.49)$ & $12.57 \pm 3.69$ & 12.49 (8.39-20.93) & 0.6757 \\
\hline Zinc (mg) & $12.64 \pm 3.49$ & $12.80(8.19-17.71)$ & $11.73 \pm 2.71$ & $10.81(8.75-17.59)$ & 0.5459 \\
\hline Cooper (mg) & $1.32 \pm 0.31$ & $1.36(0.90-1.89)$ & $1.37 \pm 0.20$ & $1.48(0.97-1.58)$ & 0.6347 \\
\hline Manganese (mg) & $5.78 \pm 2.54$ & $4.33(3.78-11.42)$ * & $5.67 \pm 1.52$ & $5.52(3.45-7.99)$ & 0.9296 \\
\hline Iodine $(\mu \mathrm{g})$ & $36.98 \pm 15.33$ & $37.99(17.68-66.31)$ & $41.19 \pm 11.98$ & $40.98(28.18-68.84)$ & 0.5253 \\
\hline Vitamin A $(\mu g)$ & $876.5 \pm 367.1$ & $1050.4(365.6-1428.1)$ & $1047.3 \pm 512.3$ & $1007.4(468.1-2219.2)$ & 0.4281 \\
\hline Vitamin D $(\mu \mathrm{g})$ & $5.59 \pm 4.28$ & $3.78(1.72-13.87) *$ & $4.42 \pm 4.05$ & $2.15(1.15-13.34)$ * & 0.8830 \\
\hline Vitamin E (mg) & $11.69 \pm 1.43$ & $11.43(9.76-14.34)$ & $12.04 \pm 4.89$ & $11.25(6.37-21.82)$ & 0.8427 \\
\hline Vitamin C (mg) & $56.09 \pm 25.69$ & $59.68(26.25-99.23)$ * & $86.50 \pm 29.35$ & 73.69 (49.42-134.77) & 0.0326 \\
\hline Vitamin B1 (mg) & $1.43 \pm 0.59$ & $1.40(0.85-2.57)$ * & $1.32 \pm 0.54$ & $1.15(0.86-2.66) *$ & 0.7911 \\
\hline Vitamin B2 (mg) & $1.76 \pm 0.51$ & $1.81(0.88-2.58)$ & $1.76 \pm 0.40$ & $1.66(1.04-2.37)$ & 0.9762 \\
\hline Vitamin B6 (mg) & $2.06 \pm 0.62$ & $2.09(1.04-2.95)$ & $2.18 \pm 0.38$ & $2.23(1.61-2.68)$ & 0.6180 \\
\hline Vitamin B12 $(\mu \mathrm{g})$ & $5.51 \pm 3.49$ & $3.92(1.34-10.27)$ * & $3.81 \pm 1.07$ & $3.98(1.72-5.22)$ & 0.1801 \\
\hline Folate $(\mu \mathrm{g})$ & $235.1 \pm 65.18$ & $243.7(114.2-325.6)$ & $263.99 \pm 53.38$ & $258.73(166.99-347.66)$ & 0.3181 \\
\hline Niacin (mg) & $23.58 \pm 7.98$ & $21.59(13.02-39.94)$ * & $20.05 \pm 5.66$ & $19.57(14.32-33.84) *$ & 0.1853 \\
\hline
\end{tabular}

${ }^{*}$ nonparametric distribution (Shapiro-Wilk test; $p \leq 0.05$ ); ${ }^{* *}$ compared while using t-Student test or Mann-Whitney test (based on distribution). 
Table 6. The nutritional value of the diet compared with the recommended values depending on the age of the female participants at baseline and after 3 months of intervention.

\begin{tabular}{|c|c|c|c|c|c|c|c|c|}
\hline \multirow{2}{*}{ Parameter } & \multirow{2}{*}{ Recommendations } & \multicolumn{3}{|c|}{ Baseline } & \multicolumn{3}{|c|}{ After 3 Months of Intervention } & \multirow{2}{*}{$p^{* * *}$} \\
\hline & & Lower & Range & Higher & Lower & Range & Higher & \\
\hline Protein & $10-15 \%$ & $0(0 \%)$ & $1(9.1 \%)$ & $10(90.9 \%)$ & $0(0 \%)$ & $1(9.1 \%)$ & $10(90.9 \%)$ & 1.0000 \\
\hline Carbohydrate & $40-70 \%$ & $8(72.7 \%)$ & $3(27.3 \%)$ & $0(0 \%)$ & $8(72.7 \%)$ & $3(27.3 \%)$ & $0(0 \%)$ & 1.0000 \\
\hline Fat & $20-35 \%$ & $0(0 \%)$ & $5(45.5 \%)$ & $6(54.5 \%)$ & $0(0 \%)$ & $6(54.5 \%)$ & $5(45.5 \%)$ & 1.0000 \\
\hline Sugars & $<10 \%$ & - & $5(45.5 \%)$ & $6(54.5 \%)$ & - & $8(72.7 \%)$ & $3(27.3 \%)$ & 0.3858 \\
\hline Cholesterol & $<300 \mathrm{mg}$ & - & $5(45.5 \%)$ & $6(54.5 \%)$ & - & $6(54.5 \%)$ & $5(45.5 \%)$ & 1.0000 \\
\hline Fiber & $>25 \mathrm{~g}$ & $8(72.7 \%)$ & $3(27.3 \%)$ & $0(0 \%)$ & $7(63.6 \%)$ & $4(36.4 \%)$ & $0(0 \%)$ & 1.0000 \\
\hline Sodium & $1200 / 1300 / 1400 / 1500 \mathrm{mg}$ * & $3(27.3 \%)$ & $0(0 \%)$ & $8(72.7 \%)$ & $5(45.5 \%)$ & $0(0 \%)$ & $6(54.5 \%)$ & 0.6576 \\
\hline Potassium & $3500 \mathrm{mg}$ * & $5(45.5 \%)$ & $0(0 \%)$ & $6(54.5 \%)$ & $6(54.5 \%)$ & $0(0 \%)$ & $5(45.5 \%)$ & 1.0000 \\
\hline Phosphorus & $580 \mathrm{mg}^{*}$ & $0(0 \%)$ & $0(0 \%)$ & $11(100 \%)$ & $0(0 \%)$ & $0(0 \%)$ & $11(100 \%)$ & 1.0000 \\
\hline Calcium & $800 / 1000 \mathrm{mg} *$ & $10(90.9 \%)$ & $0(0 \%)$ & $1(9.1 \%)$ & $11(100 \%)$ & $0(0 \%)$ & $0(0 \%)$ & 1.0000 \\
\hline Magnesium & $265 \mathrm{mg}$ * & $3(27.3 \%)$ & $0(0 \%)$ & $8(72.7 \%)$ & $2(18.2 \%)$ & $0(0 \%)$ & $9(81.8 \%)$ & 1.0000 \\
\hline Iron & $6 / 8 \mathrm{mg} *$ & $0(0 \%)$ & $0(0 \%)$ & $11(100 \%)$ & $0(0 \%)$ & $0(0 \%)$ & $11(100 \%)$ & 1.0000 \\
\hline Zinc & $6.8 \mathrm{mg}^{*}$ & $2(18.2 \%)$ & $0(0 \%)$ & $9(81.8 \%)$ & $1(9.1 \%)$ & $0(0 \%)$ & $10(90.9 \%)$ & 1.0000 \\
\hline Cooper & $0.7 \mathrm{mg}$ * & $1(9.1 \%)$ & $0(0 \%)$ & $10(90.9 \%)$ & $1(9.1 \%)$ & $0(0 \%)$ & $10(90.9 \%)$ & 1.0000 \\
\hline Manganese & $1.8 \mathrm{mg}^{* *}$ & $1(9.1 \%)$ & $0(0 \%)$ & $10(90.9 \%)$ & $1(9.1 \%)$ & $0(0 \%)$ & $10(90.9 \%)$ & 1.0000 \\
\hline Iodine & $95 \mu \mathrm{g} *$ & $11(100 \%)$ & $0(0 \%)$ & $0(0 \%)$ & $11(100 \%)$ & $0(0 \%)$ & $0(0 \%)$ & 1.0000 \\
\hline Vitamin A & $500 \mu \mathrm{g}$ * & $2(18.2 \%)$ & $0(0 \%)$ & $9(81.8 \%)$ & $0(0 \%)$ & $0(0 \%)$ & $11(100 \%)$ & 0.4583 \\
\hline Vitamin D & $15 \mu \mathrm{g} * *$ & $11(100 \%)$ & $0(0 \%)$ & $0(0 \%)$ & $11(100 \%)$ & $0(0 \%)$ & $0(0 \%)$ & 1.0000 \\
\hline Vitamin E & $8 \mathrm{mg}^{* *}$ & $3(27.3 \%)$ & $0(0 \%)$ & $8(72.7 \%)$ & $0(0 \%)$ & $0(0 \%)$ & $11(100 \%)$ & 0.2140 \\
\hline Vitamin C & $60 \mathrm{mg}$ * & $3(27.3 \%)$ & $0(0 \%)$ & $8(72.7 \%)$ & $3(27.3 \%)$ & $0(0 \%)$ & $8(72.7 \%)$ & 1.0000 \\
\hline Vitamin B1 & $0.9 \mathrm{mg}$ * & $3(27.3 \%)$ & $0(0 \%)$ & $8(72.7 \%)$ & $3(27.3 \%)$ & $0(0 \%)$ & $8(72.7 \%)$ & 1.0000 \\
\hline Vitamin B2 & $0.9 \mathrm{mg}$ * & $0(0 \%)$ & $0(0 \%)$ & $11(100 \%)$ & $0(0 \%)$ & $0(0 \%)$ & $11(100 \%)$ & 1.0000 \\
\hline Vitamin B6 & $1.1 / 1.3 \mathrm{mg}^{*}$ & $2(18.2 \%)$ & $0(0 \%)$ & $9(81.8 \%)$ & $2(18.2 \%)$ & $0(0 \%)$ & $9(81.8 \%)$ & 1.0000 \\
\hline Vitamin B12 & $2.0 \mu \mathrm{g} *$ & $1(9.1 \%)$ & $0(0 \%)$ & $10(90.9 \%)$ & $0(0 \%)$ & $0(0 \%)$ & $11(100 \%)$ & 1.0000 \\
\hline Folate & $320 \mu \mathrm{g}$ * & $10(90.9 \%)$ & $0(0 \%)$ & $1(9.1 \%)$ & $10(90.9 \%)$ & $0(0 \%)$ & $1(9.1 \%)$ & 1.0000 \\
\hline Niacin & $11 \mathrm{mg} *$ & $0(0 \%)$ & $0(0 \%)$ & $11(100 \%)$ & $1(9.1 \%)$ & $0(0 \%)$ & $10(90.9 \%)$ & 1.0000 \\
\hline
\end{tabular}

${ }^{*}$ EAR—estimated average requirement; ${ }^{* *} \mathrm{AI}$-adequate intake; ${ }^{* * *}$ compared while using chi $^{2}$ test.

The nutritional value of the diet compared with the recommended values depending on the age of the male participants at baseline and after 3 months of intervention is presented in Table 7. It was observed that all the parameters were comparable before and after intervention, and no statistically significant differences were observed for male participants $(p>0.05)$. The majority of participating men consumed excessive amounts of protein, cholesterol, sodium, phosphorus, iron, zinc, copper and manganese, as well as vitamins A, E, B1, B2, B6 and B12 and niacin. They also consumed insufficient amounts of carbohydrates, fiber, magnesium, calcium, iodine, vitamins $\mathrm{D}$ and $\mathrm{C}$ and folate.

The atherogenic index of Keys was also comparable for the baseline diet and the diet after 3 months of intervention, both for females $(71.98 \pm 14.79$ vs. $71.58 \pm 16.68$; $p=0.9530$; t-Student test) and for males $(75.39 \pm 24.87$ vs. $78.92 \pm 29.89 ; p=0.4799$; Mann-Whitney test).

The basic physical activity parameters of the female participants at baseline and after 3 months of intervention are presented in Table 8. It was observed that all the parameters were comparable before and after intervention, and no statistically significant differences were observed for female participants $(p>0.05)$. The additional analyses of the basic physical activity parameters of the participants after 3 months of intervention compared between sub-groups following recommendations and not following dietary and physical activity recommendations, respectively, are presented in the Supplementary Materials (Tables S12 and S13, respectively), and they indicated differences for energy expenditure, physical activity duration, active energy expenditure, number of steps and average METs. Similarly, analyses of the basic physical activity parameters of the female participants not following dietary recommendations and following dietary recommendations, respectively, at baseline and after 3 months of intervention, are presented in the Supplementary Materials (Tables S14 and S15, respectively), and they are in agreement with the results for the total studied group. 
Table 7. The nutritional value of the diet compared with the recommended values depending on the age of the male participants at baseline and after 3 months of intervention.

\begin{tabular}{|c|c|c|c|c|c|c|c|c|}
\hline \multirow{2}{*}{ Parameter } & \multirow{2}{*}{ Recommendations } & \multicolumn{3}{|c|}{ Baseline } & \multicolumn{3}{|c|}{ After 3 Months of Intervention } & \multirow{2}{*}{$p^{* * *}$} \\
\hline & & Lower & Range & Higher & Lower & Range & Higher & \\
\hline Protein & $10-15 \%$ & $0(0 \%)$ & $3(33.3 \%)$ & $6(66.7 \%)$ & $1(11.1 \%)$ & $0(0 \%)$ & $8(88.9 \%)$ & 0.1173 \\
\hline Carbohydrate & $40-70 \%$ & $6(66.7 \%)$ & $3(33.3 \%)$ & $0(0 \%)$ & $7(77.8 \%)$ & $2(22.2 \%)$ & $0(0 \%)$ & 1.0000 \\
\hline Fat & $20-35 \%$ & $0(0 \%)$ & $5(55.6 \%)$ & $4(44.4 \%)$ & - & $3(33.3 \%)$ & $6(66.7 \%)$ & 0.6353 \\
\hline Sugars & $<10 \%$ & - & $3(33.3 \%)$ & $6(66.7 \%)$ & - & $6(66.7 \%)$ & $3(33.3 \%)$ & 0.3458 \\
\hline Cholesterol & $<300 \mathrm{mg}$ & - & $2(22.3 \%)$ & $7(77.8 \%)$ & - & $3(33.3 \%)$ & $6(66.7 \%)$ & 1.0000 \\
\hline Fiber & $>25 \mathrm{~g}$ & $6(66.7 \%)$ & $3(33.3 \%)$ & $0(0 \%)$ & $6(66.7 \%)$ & $3(33.3 \%)$ & $0(0 \%)$ & 1.0000 \\
\hline Sodium & $1400 / 1500 \mathrm{mg}$ * & $0(0 \%)$ & $0(0 \%)$ & $9(100 \%)$ & $3(33.3 \%)$ & $0(0 \%)$ & $6(66.7 \%)$ & 0.2059 \\
\hline Potassium & $3500 \mathrm{mg}$ * & $3(33.3 \%)$ & $0(0 \%)$ & $6(66.7 \%)$ & $1(11.1 \%)$ & $0(0 \%)$ & $8(88.9 \%)$ & 0.5707 \\
\hline Phosphorus & $580 \mathrm{mg}$ * & $0(0 \%)$ & $0(0 \%)$ & $9(100 \%)$ & $0(0 \%)$ & $0(0 \%)$ & $9(100 \%)$ & 1.0000 \\
\hline Calcium & $800 / 1000 \mathrm{mg}$ * & $8(88.9 \%)$ & $0(0 \%)$ & $1(11.1 \%)$ & $9(100 \%)$ & $0(0 \%)$ & $0(0 \%)$ & 1.0000 \\
\hline Magnesium & $350 \mathrm{mg}$ * & $6(66.7 \%)$ & $0(0 \%)$ & $3(33.3 \%)$ & $6(66.7 \%)$ & $0(0 \%)$ & $3(33.3 \%)$ & 1.0000 \\
\hline Iron & $6 \mathrm{mg}$ * & $0(0 \%)$ & $0(0 \%)$ & $9(100 \%)$ & $0(0 \%)$ & $0(0 \%)$ & $9(100 \%)$ & 1.0000 \\
\hline Zinc & $9.4 \mathrm{mg}$ * & $2(22.2 \%)$ & $0(0 \%)$ & $7(77.8 \%)$ & $0(0 \%)$ & $1(11.1 \%)$ & $8(88.9 \%)$ & 1.0000 \\
\hline Cooper & $0.7 \mathrm{mg}$ * & $0(0 \%)$ & $0(0 \%)$ & $9(100 \%)$ & $0(0 \%)$ & $0(0 \%)$ & $9(100 \%)$ & 1.0000 \\
\hline Manganese & $2.3 \mathrm{mg}^{* *}$ & $0(0 \%)$ & $0(0 \%)$ & $9(100 \%)$ & $0(0 \%)$ & $0(0 \%)$ & $9(100 \%)$ & 1.0000 \\
\hline Iodine & $95 \mu \mathrm{g} *$ & $9(100 \%)$ & $0(0 \%)$ & $0(0 \%)$ & $9(100 \%)$ & $0(0 \%)$ & $0(0 \%)$ & 1.0000 \\
\hline Vitamin A & $630 \mu \mathrm{g} *$ & $4(44.4 \%)$ & $0(0 \%)$ & $5(55.6 \%)$ & $0(0 \%)$ & $0(0 \%)$ & $9(100 \%)$ & 0.0890 \\
\hline Vitamin D & $15 \mu \mathrm{g} * *$ & $9(100 \%)$ & $0(0 \%)$ & $0(0 \%)$ & $9(100 \%)$ & $0(0 \%)$ & $0(0 \%)$ & 1.0000 \\
\hline Vitamin E & $10 \mathrm{mg}$ ** & $1(11.1 \%)$ & $0(0 \%)$ & $8(88.9 \%)$ & $3(33.3 \%)$ & $0(0 \%)$ & $6(66.7 \%)$ & 0.5707 \\
\hline Vitamin C & 75 mg * & $7(77.8 \%)$ & $0(0 \%)$ & $2(22.2 \%)$ & $5(55.6 \%)$ & $0(0 \%)$ & $4(44.4 \%)$ & 0.6171 \\
\hline Vitamin B1 & $1.1 \mathrm{mg}$ * & $4(44.4 \%)$ & $0(0 \%)$ & $5(55.6 \%)$ & $3(33.3 \%)$ & $0(0 \%)$ & $6(66.7 \%)$ & 1.0000 \\
\hline Vitamin B2 & $1.1 \mathrm{mg}$ * & $1(11.1 \%)$ & $0(0 \%)$ & $8(88.9 \%)$ & $1(11.1 \%)$ & $0(0 \%)$ & $8(88.9 \%)$ & 1.0000 \\
\hline Vitamin B6 & $1.1 / 1.4 \mathrm{mg}$ * & $1(11.1 \%)$ & $0(0 \%)$ & $8(88.9 \%)$ & $0(0 \%)$ & $0(0 \%)$ & $9(100 \%)$ & 1.0000 \\
\hline Vitamin B12 & $2.0 \mu \mathrm{g} *$ & $2(22.2 \%)$ & $0(0 \%)$ & $7(77.8 \%)$ & $1(11.1 \%)$ & $0(0 \%)$ & $8(88.9 \%)$ & 1.0000 \\
\hline Folate & $320 \mu \mathrm{g}$ * & $8(88.9 \%)$ & $0(0 \%)$ & $1(11.1 \%)$ & $7(77.8 \%)$ & $0(0 \%)$ & $2(22.2 \%)$ & 1.0000 \\
\hline Niacin & $12 \mathrm{mg}$ * & $0(0 \%)$ & $0(0 \%)$ & $9(100 \%)$ & $0(0 \%)$ & $0(0 \%)$ & $9(100 \%)$ & 1.0000 \\
\hline
\end{tabular}

*EAR—estimated average requirement; ${ }^{* *} \mathrm{AI}$-adequate intake; ${ }^{* * *}$ compared while using chi $^{2}$ test.

Table 8. The basic physical activity parameters of the female participants at baseline and after 3 months of intervention.

\begin{tabular}{|c|c|c|c|c|c|}
\hline \multirow{2}{*}{ Parameter } & \multicolumn{2}{|c|}{ Baseline } & \multicolumn{2}{|c|}{ After 3 Months of Intervention } & \multirow{2}{*}{$p^{* *}$} \\
\hline & Mean \pm SD & Median (Min-Max) & Mean \pm SD & Median (Min-Max) & \\
\hline Total energy expenditure (kcal) & $1293.9 \pm 170.9$ & $1283.0(1066.0-1601.0)$ & $1401.9 \pm 269.8$ & 1308.5 (1090.0-1955.5) & 0.2819 \\
\hline Physical activity duration (min) & $27.7 \pm 16.7$ & $25.3(10.7-69.0)^{*}$ & $39.5 \pm 32.4$ & $28.2(10.7-122.7) *$ & 0.3981 \\
\hline Lying down duration (min) & $220.9 \pm 57.0$ & $207.0(112.7-334.7)$ & $236.3 \pm 80.9$ & $244.0(82.3-362.0)$ & 0.6181 \\
\hline Active energy expenditure (kcal) & $173.3 \pm 98.4$ & $152.3(59.7-404.3)$ & $246.6 \pm 186.3$ & $183.7(62.3-703.0) *$ & 0.2751 \\
\hline Number of steps & $4599 \pm 1839$ & $3867(2945-8948) *$ & $5471 \pm 2409$ & $5276(2271-10964)$ & 0.2751 \\
\hline Sleep duration (min) & $179.3 \pm 56.2$ & $181.7(89.3-295.3)$ & $186.4 \pm 75.3$ & $200.8(55.0-295.0)$ & 0.8097 \\
\hline Average METs & $1.08 \pm 0.16$ & $1.07(0.87-1.43)$ & $1.20 \pm 0.28$ & $1.12(0.93-1.87)$ & 0.2248 \\
\hline
\end{tabular}

MET-metabolic equivalent of task; * nonparametric distribution (Shapiro-Wilk test; $p \leq 0.05$ ); ${ }^{* *}$ compared while using $\mathrm{t}$-Student test or

Mann-Whitney test (based on distribution).

The basic physical activity parameters of the male participants at baseline and after 3 months of intervention are presented in Table 9. It was observed that the majority of parameters were comparable before and after intervention, and no statistically significant differences were observed for male participants $(p>0.05)$. Only metabolic equivalents of tasks (METs) increased (1.02 \pm 0.41 vs. $1.34 \pm 0.12 ; p<0.0001)$. The additional analyses of the basic physical activity parameters of the male participants not following dietary recommendations and following dietary recommendations, respectively, at baseline and after 3 months of intervention, are presented in the Supplementary Materials (Tables S16 and S17, respectively), and they are in general agreement with the results for the total studied group.

The biochemical parameters of the participants at baseline and after 3 months of intervention are presented in Table 10. It was observed that blood triglyceride levels (140.36 \pm 14.92 vs. $110.04 \pm 37.23 \mathrm{mg} / \mathrm{dL} ; p=0.0017)$ and gamma-glutamyltransferase (GGT) activity decreased ( $23.03 \pm 12.87$ vs. $12.77 \pm 5.24 \mathrm{U} / \mathrm{L} ; p=0.0008)$, but the other parameters did not differ $(p>0.05)$. The additional analyses of the biochemical parameters of the participants after 3 months of intervention compared between sub-groups following 
recommendations and not following recommendations are presented in the Supplementary Materials (Table S18), and they indicated differences for total cholesterol and LDL cholesterol levels. Similarly, analyses of the biochemical parameters of the female participants not following dietary recommendations and following dietary recommendations, respectively, at baseline and after 3 months of intervention, are presented in the Supplementary Materials (Tables S19 and S20, respectively), while the analyses of the biochemical parameters of the male participants not following dietary recommendations and following dietary recommendations, respectively, at baseline and after 3 months of intervention, are presented in the Supplementary Materials (Tables S21 and S22, respectively), and they indicated differences for GGT levels in female participants following dietary recommendations and for male participants following dietary recommendations, as well as for triglyceride levels for male participants not following dietary recommendations. At the same time, the analyses of the biochemical parameters after 3 months of intervention compared between sub-groups based on gender, age and body mass status are presented in the Supplementary Materials (Table S23), and they indicated differences for GGT and ALT levels.

Table 9. The basic physical activity parameters of the male participants at baseline and after 3 months of intervention.

\begin{tabular}{|c|c|c|c|c|c|}
\hline \multirow{2}{*}{ Parameter } & \multicolumn{2}{|c|}{ Baseline } & \multicolumn{2}{|c|}{ After 3 Months of Intervention } & \multirow{2}{*}{$p^{* *}$} \\
\hline & Mean \pm SD & Median (Min-Max) & Mean \pm SD & Median (Min-Max) & \\
\hline Total energy expenditure (kcal) & $1617.9 \pm 431.9$ & $1522.0(1266.3-2704.0)$ * & $1624.6 \pm 239.5$ & $1628.0(1250.7-1931.5)$ & 0.5317 \\
\hline Physical activity duration (min) & $55.7 \pm 73.4$ & $38.7(10.7-247.7) *$ & $50.2 \pm 13.9$ & $52.7(30.7-70.0)$ & 0.1358 \\
\hline Lying down duration (min) & $211.4 \pm 37.2$ & $227.3(154.3-248.0) *$ & $220.7 \pm 68.9$ & $207.0(110.3-345.0)$ & 0.8099 \\
\hline Active energy expenditure (kcal) & $379.4 \pm 509.0$ & $281.3(58.0-1705.7) *$ & $327.9 \pm 100.5$ & $324.3(181.0-474.3)$ & 0.2685 \\
\hline Number of steps & $4482 \pm 2033$ & $3785(2380-8065)$ & $5512 \pm 2038$ & $5792(2356-8326)$ & 0.3142 \\
\hline Sleep duration (min) & $168.9 \pm 34.0$ & $159.3(112.0-224.0)$ & $170.0 \pm 48.9$ & $165.5(81.3-255.0)$ & 0.9568 \\
\hline Average METs & $1.02 \pm 0.41$ & $1.00(0.87-1.17)$ & $1.34 \pm 0.12$ & $1.35(1.10-1.47)$ & $<0.0001$ \\
\hline
\end{tabular}

MET-metabolic equivalent of task; ${ }^{*}$ nonparametric distribution (Shapiro-Wilk test; $p \leq 0.05$ ); ${ }^{* *}$ compared while using t-Student test or Mann-Whitney test (based on distribution).

Table 10. The biochemical parameters of the participants at baseline and after 3 months of intervention.

\begin{tabular}{|c|c|c|c|c|c|}
\hline \multirow{2}{*}{ Parameter } & \multicolumn{2}{|c|}{ Baseline } & \multicolumn{2}{|c|}{ After 3 Months of Intervention } & \multirow{2}{*}{$p^{* *}$} \\
\hline & Mean \pm SD & Median (Min-Max) & Mean \pm SD & Median (Min-Max) & \\
\hline Triglycerides (mg/dL) & $140.36 \pm 14.92$ & 138.95 (116.98-185.52) & $110.04 \pm 37.23$ & $127.26(64.03-166.99)$ * & 0.0017 \\
\hline Total cholesterol (mg/dL) & $153.73 \pm 39.94$ & $143.61(105.73-252.26)$ & $145.78 \pm 39.74$ & 135.72 (91.24-234.65) & 0.5374 \\
\hline HDL-C (mg/dL) & $32.27 \pm 11.13$ & $30.01(19.56-66.66) *$ & $29.73 \pm 7.56$ & $28.11(18.11-50.23)$ & 0.5457 \\
\hline LDL-C (mg/dL) & $93.38 \pm 35.15$ & $88.99(40.21-171.34)$ & $94.05 \pm 37.02$ & 82.99 (51.3-179.70) & 0.9544 \\
\hline ALT (U/L) & $11.36 \pm 5.78$ & $11.90(2.09-21.59)$ & $13.66 \pm 6.43$ & $13.21(7.33-34.48) *$ & 0.4857 \\
\hline GGT (U/L) & $23.03 \pm 12.87$ & $20.28(9.18-58.75) *$ & $12.77 \pm 5.24$ & $11.55(7.00-27.89)$ * & 0.0008 \\
\hline
\end{tabular}

HDL-C—high-density lipoprotein cholesterol; LDL-C—low-density lipoprotein cholesterol; ALT—alanine aminotransferase; GGT—gammaglutamyltransferase; ${ }^{*}$ nonparametric distribution (Shapiro-Wilk test; $p \leq 0.05$ ); ${ }^{* *}$ compared while using t-Student test or Mann-Whitney test (based on distribution).

\section{Discussion}

The dietary management of NAFLD is associated mainly with a low-energy diet, as body mass reduction is the most effective way to reduce liver steatosis [47]. It is indicated that a body mass reduction of $7-10 \%$ resolves steatohepatitis and improves fibrosis, confirmed by liver histopathology [36,48]. In this conducted study, body mass reduction was not observed in spite of the fact that patients received the recommendation of a low-energy diet, which was encouraged to be followed every 2 weeks. However, it should be emphasized that body mass reduction is a long-term process, and, in the case of NAFLD patients, it is commonly unsuccessful [49]. As a result, the risk of fibrosis caused by NASH progression still exists [50].

Within the possible reasons of unsuccessful body mass reduction, caused by not following the low-energy diet, there may be an association with socializing and spending time with friends. This results from the fact that the study was conducted in the period of spring and summer, which is typically associated with barbecue socializing, indicated 
to be a serious health risk factor [51]. Other authors also observed body mass increase during weekends [52], as well as holidays [53], associated with people quitting diets in those periods. Moreover, such body mass increases after weekends or holidays may not be corrected in the following period [54], resulting in a cumulative body mass increase over years. Even in the case of patients undergoing a body mass reduction, such a body mass increase is observed in holiday periods [55]; therefore, during this period, it should be planned to include any additional elements of therapy (such as phone and e-mail counselling [56]) to motivate patients to follow their diet. This is associated with the fact that the observed body mass increase during the indicated periods results mainly from the increased intake of food accompanied by a weakened satiety response, not from reduced energy expenditure [57].

In the case of obese patients, both individual and group diet therapy may be used to reduce excessive body mass [58]. In the presented study, individual dietary therapy was chosen in order to provide individual recommendations for each participant and to provide the possibility of individual correction of the diet and to motivate patients. However, as indicated above, the applied model for some patients was not effective, which was proposed to be caused by their participation in socializing and the associated additional consumption of food. It may be supposed that increased effectiveness may have been obtained by including additional behavioral therapy, which is effective in supporting body mass reduction [59]. At the same time, the duration of the applied intervention may have been too short to obtain effective changes in dietary and physical activity habits, which may be supposed based on the suggestions by other authors indicating that body mass reduction therapy should be planned for 10 months or even 18 months to increase its effectiveness [60]. Moreover, group therapy may have been a better approach for this group, as some studies suggest that group therapy is characterized by a higher effectiveness even for patients who declare that they prefer to participate in individual therapy [61]. Taking this into account, further studies should be planned also with a model of group therapy, including behavioral therapy, and planned for a longer time to verify the potential influence of various models of therapy on body mass reduction in NAFLD patients.

In spite of the fact that no effective body mass reduction and no change in diet were observed in the conducted study, some biochemical parameters after 3 months of the experiment changed. This may be attributed to the milk thistle seed supply, which as a source of silymarin, may have caused a change in GGT levels. Silymarin extract is known to cause an effective decrease in ALT and aspartate aminotransferase (AST) blood levels in NAFLD patients, which is confirmed by the meta-analysis by Zhong et al. [62]. In the study by Cacciapuoti et al. [30], in a group of patients receiving silymarin extract (in tablets) for 6 weeks twice a day, it was observed that their ALT, AST and GGT blood levels decreased. While in the present study, GGT activity decreased, but ALT did not change; it may be supposed that either the doses were too low or the intervention was too short to induce such effects. However, the observed results are promising and should be analyzed in the future.

It should be emphasized that in the studies published to date, mainly milk thistle seed extract was studied; therefore, it may be supposed that the effect observed in the present study may be incomparable, and it may also be attributed to other components of milk thistle seeds. Taking this into account, the effects of milk thistle seeds, extract and silymarin should be compared in further research. Moreover, it should be hypothesized that depending on the agricultural conditions, the composition of seeds may differ, which may also influence the observed results. However, while analyzing the literature, it must be noted that milk thistle seeds contain linoleic acid and oleic acid and also $\alpha$-tocopherol and phenolic acids, such as vanillic acid, p-coumaric acid and silybin [63]. Therefore, it may be supposed that the effect observed in the current study may be attributed to various components including p-coumaric acid, which is an antioxidative and anti-inflammatory component, as it was stated by Amalan et al. [64] that it may modulate lipid metabolism, reducing observed disturbances. Similarly, in the study by Cha et al. [65], it was observed 
that $\mathrm{p}$-coumaric acid improves triglyceride levels in the blood of rats after myocardial infarction. In another study, phenolic acids, from berries, including p-coumaric acid, were effective in reducing triglyceride accumulation in hepatocytes in the early stage of NAFLD [66].

In the current study, the triglyceride levels, after the experiment, were significantly lower than at baseline. This may be compared with the results of the study by Huseini et al. [21], as in their study, the group with type 2 diabetes mellitus received $200 \mathrm{mg}$ of silymarin in the form of tablets 3 times a day, which caused a decrease in total cholesterol, LDL cholesterol, triglycerides and liver enzyme blood levels after 4 months of treatment. Similarly, in the study by Famouri et al. [67], conducted on a group of children aged 5-16 with NAFLD, using $5 \mathrm{mg}$ of silymarin per $\mathrm{kg}$ of body mass (in the form of tablets) that was combined with lifestyle modifications for 12 weeks, triglyceride and liver enzyme blood levels were corrected, while neither LDL nor HDL cholesterol blood level changed. Such results are similar as those presented in the current study, and it may be supposed that triglyceride blood levels may be more prone to change after silymarin supply than cholesterol blood levels. In the current study, the applied daily dose of milk thistle seeds provided $210 \mathrm{mg}$ of silymarin, while in the other studies, higher doses were applied to provide $280 \mathrm{mg}$ of silymarin [68-71], $1260 \mathrm{mg}$ of silymarin [72] or $2100 \mathrm{mg}$ of silymarin [72,73]. Moreover, it should be noted that while the above-mentioned studies by Huseini et al. [21] and Famouri et al. [67] presented interventions based on silymarin extracts, the current study revealed that milk thistle seeds included in the diet may also be effective. It needs to be emphasized that some studies indicated, similarly to the current study, improved biochemical parameters of patients using silymarin even if their body mass reduction was not obtained, as it was observed by Kalopitas [74] for transaminase levels based on a number of studies $[68,69,73,75]$. In the current study, no statistically significant effect on ALT level was observed, but at the same time, GGT and TG levels were significantly lower in spite of there being no significant reduction of body mass. This may have been caused by dietary changes that were associated with a choice of products that were different to those previously chosen, but that did not contribute to a lower energy value of diet, thereby not causing body mass reduction; however, when accompanied by silymarin supply, they may have supported the correction of some biochemical parameters.

In spite of the fact that patients in the current study received specific dietary recommendations, their diet at baseline and after 3 months of treatment were comparable- - the significant differences were observed neither for the nutritional value of the diet nor for the share of the group following the specific dietary recommendations. While comparing the diet that was followed by the participants of the current study with the general recommendations [45], as well as with the dietary recommendations for NAFLD patients undergoing body mass reduction [36,37], it should be noted that in the studied group, there were some serious problems. The most important was the very low fiber supply, which was not improved within the experiment, as increased fiber intake is recommended both during body mass reduction and for NAFLD patients [76].

The liver plays a crucial role in the metabolism of minerals, which may be disturbed in the case of chronic liver diseases, but at the same time, excessive or insufficient supply of minerals may increase disturbances of homeostasis [77]. In the studied group, it was observed that the common problems were excessive supplies of sodium, phosphorus and iron, accompanied by insufficient supplies of potassium, magnesium, calcium and zinc. Similar results were observed by Da Silva et al. [78] in a group of patients with NAFLD, as they observed excessive supplies of sodium, iron and zinc, accompanied by insufficient supplies of magnesium and calcium. The described results indicate that there is a serious need for change in the dietary behaviors of NAFLD patients, as sodium intake is positively correlated with NAFLD progression, and this disease is more common in cases of individuals characterized by an excessive intake of this nutrient than in cases of the other ones $[79,80]$. Sodium intake should be reduced by the limitation of processed food 
consumption, and it should be accompanied by an increased intake of potassium, which as a sodium antagonist, may be obtained by increased fruit and vegetable consumption [81].

The phosphorus intake in the studied group was also excessive, but while it is proven that its level may be harmful, little is known about the direct influence of its excessive dietary intake [82]. At the same time, insufficient magnesium intake observed in the studied group of NAFLD patients is a common problem in patients with liver steatosis, while insufficient magnesium intake may promote inflammation and insulin resistance in this group, and increased intake reduces the risk [83], but excessive intake may also have a negative impact, including the increased risk of death for patients with liver steatosis [84]. Similarly, it was noted that low zinc intake is a common problem in patients with liver diseases, which may also lead to insulin resistance and promote liver steatosis; therefore, it is recommended that NAFLD patients apply adequate supplementation of this mineral [85].

In the case of vitamins, in the studied group it was observed that the common problems were excessive supplies of vitamins A, E, B1, B2, B6 and B12 and niacin, accompanied by insufficient supplies of vitamin $\mathrm{D}$ and folate. Similar results were observed in the above-mentioned study by Da Silva et al. [78] in a group of patients with NAFLD, as they also found low vitamin $D$ intake. This problem is not only common in NAFLD patients, but a low vitamin D level is associated with NAFLD progression; thus, vitamin D supplementation may relieve NAFLD symptoms, especially those associated with inflammation [86]. At the same time, an excessive supply of B vitamins is not a problem, as it is removed from the body with urine [87].

The general quality of the diet is reversely associated with NAFLD progression [88], and a higher quality diet may decrease the risk of the disease, as well as be beneficial for patients with already diagnosed NAFLD [89]. However, the diet of the studied patients was not corrected within the study, and that corresponds with the atherogenic index of Keys, which did not change within the study and which may suggest a high atherogenic potential of the diet. When combined with a low fiber intake, this situation may promote the development of cardiovascular diseases [90]. Taking this into account, it seems necessary to promote changes in diet and lifestyle in a more effective way to a group of NAFLD patients, as well as to provide them with adequate counselling and support their motivation. It may be supposed that inadequate motivation was a key problem in the studied group, as in spite of the fact that participants were supervised, to follow recommendations, they did not follow them but only applied the milk thistle. This is confirmed by other authors indicating that motivation and engagement is necessary for a proper body mass reduction [91]; thus, not only should a physician and dietitian have been planned to participate in the therapy, but also a psychologist [92].

\section{Conclusions}

As the diet and physical activity of the studied patients were not changed within the studied period, it may be supposed that the beneficial decrease in blood triglyceride levels and GGT activity was obtained due to the milk thistle supply as a source of silymarin. It may be indicated that including milk thistle in an everyday diet may be an additional recommendation for NAFLD patients, which allows one to obtain beneficial effects, even if the other recommendations are not followed.

Supplementary Materials: The following are available online at https:/ / www.mdpi.com/article/ 10.3390/app11135836/s1, Table S1: The body mass and body composition of the participants after 3 months of intervention compared between sub-groups following recommendations and not following recommendations, Table S2: The body mass and body composition of the female participants not following dietary recommendations at baseline and after 3 months of intervention, Table S3: The body mass and body composition of the female participants following dietary recommendations at baseline and after 3 months of intervention, Table S4: The body mass and body composition of the male participants not following dietary recommendations at baseline and after 3 months of intervention, Table S5: The body mass and body composition of the male participants following dietary recommendations at baseline and after 3 months of intervention, Table S6: The energy and 
nutritional value of the diet of the participants after 3 months of intervention compared between sub-groups following recommendations and not following dietary recommendations, Table S7: The energy and nutritional value of the diet of the participants after 3 months of intervention compared between sub-groups following recommendations and not following physical activity recommendations, Table S8: The energy and nutritional value of the diet of the female participants not following dietary recommendations at baseline and after 3 months of intervention, Table S9: The energy and nutritional value of the diet of the female participants following dietary recommendations at baseline and after 3 months of intervention, Table S10: The energy and nutritional value of the diet of the male participants not following dietary recommendations at baseline and after 3 months of intervention, Table S11;The energy and nutritional value of the diet of the male participants following dietary recommendations at baseline and after 3 months of intervention, Table S12: The basic physical activity parameters of the participants after 3 months of intervention compared between sub-groups following recommendations and not following dietary recommendations, Table S13: The basic physical activity parameters of the participants after 3 months of intervention compared between sub-groups following recommendations and not following physical activity recommendations, Table S14: The basic physical activity parameters of the female participants not following dietary recommendations at baseline and after 3 months of intervention, Table S15: The basic physical activity parameters of the female participants following dietary recommendations at baseline and after 3 months of intervention, Table S16: The basic physical activity parameters of the male participants not following dietary recommendations at baseline and after 3 months of intervention, Table S17:The basic physical activity parameters of the male participants following dietary recommendations at baseline and after 3 months of intervention, Table S18: The biochemical parameters of the participants after 3 months of intervention compared between sub-groups following recommendations and not following recommendations, Table S19: The biochemical parameters of the female participants not following dietary recommendations at baseline and after 3 months of intervention, Table S20: The biochemical parameters of the female participants following dietary recommendations at baseline and after 3 months of intervention, Table S21: The biochemical parameters of the male participants not following dietary recommendations at baseline and after 3 months of intervention, Table S22: The biochemical parameters of the male participants following dietary recommendations at baseline and after 3 months of intervention, Table S23: The biochemical parameters after 3 months of intervention compared between sub-groups based on gender, age and body mass status.

Author Contributions: Conceptualization, A.K.; methodology, A.K.; formal analysis, A.K.; investigation, A.K.; writing—original draft preparation, A.K. and D.G.; writing—review and editing, A.K. and D.G. All authors have read and agreed to the published version of the manuscript.

Funding: This research was funded by the Polish Ministry of Science and Higher Education grant (WULS-SGGW: 505-10-100400-M00295-99/2015).

Institutional Review Board Statement: The study was conducted according to the guidelines of the Declaration of Helsinki and approved by the Ethics Committee of the Central Clinical Hospital of the Ministry of Interior in Warsaw (9/2016). Written informed consent was provided by all participants of the study.

Informed Consent Statement: Informed consent was obtained from all subjects involved in the study.

Data Availability Statement: Not applicable.

Conflicts of Interest: The authors declare no conflict of interest.

\section{References}

1. Asrani, S.K.; Devarbhavi, H.; Eaton, J.; Kamath, P.S. Burden of liver diseases in the world. J. Hepatol. 2019, 70, 151-171. [CrossRef]

2. Younossi, Z.M.; Marchesini, G.; Pinto-Cortez, H.; Petta, S. Epidemiology of nonalcoholic fatty liver disease and nonalcoholic steatohepatitis: Implications for liver transplantation. Transplantation 2019, 103, 22-27. [CrossRef] [PubMed]

3. Chalasani, N.; Younossi, Z.; Lavine, J.E.; Charlton, M.; Cusi, K.; Rinella, M.; Harrison, S.A.; Brunt, E.M.; Sanyal, A.J. The diagnosis and management of nonalcoholic fatty liver disease: Practice guidance from the American Association for the Study of Liver Diseases. Hepatology 2018, 67, 328-357. [CrossRef]

4. Ore, A.; Akinloye, O.A. Oxidative stress and antioxidant biomarkers in clinical and experimental models of non-alcoholic fatty liver disease. Medicina 2019, 55, 26. [CrossRef] 
5. Younossi, Z.M. Non-alcoholic fatty liver disease-A global public health perspective. J. Hepatol. 2019, 70, 531-544. [CrossRef]

6. Massoud, O.; Charlton, M. Nonalcoholic fatty liver disease/nonalcoholic steatohepatitis and hepatocellular carcinoma. Clin. Liver Dis. 2018, 22, 201-211. [CrossRef]

7. Marchisello, S.; Di Pino, A.; Scicali, R.; Urbano, F.; Piro, S.; Purrello, F.; Rabuazzo, A.M. Pathophysiological, molecular and therapeutic issues of nonalcoholic fatty liver disease: An overview. Int. J. Mol. Sci. 2019, 20, 1948. [CrossRef]

8. Ryoo, J.H.; Suh, Y.J.; Shin, H.C.; Cho, Y.K.; Choi, J.M.; Park, S.K. Clinical association between non-alcoholic fatty liver disease and the development of hypertension. J. Gastroenterol. Hepatol. 2014, 29, 1926-1931. [CrossRef]

9. Fotbolcu, H.; Zorlu, E. Nonalcoholic fatty liver disease as a multi-systemic disease. World J. Gastroenterol. 2016, 22, 4079-4090. [CrossRef] [PubMed]

10. Oikonomou, D.; Georgiopoulos, G.; Katsi, V.; Kourek, C.; Tsioufis, C.; Alexopoulou, A.; Koutli, E.; Tousoulis, D. Non-alcoholic fatty liver disease and hypertension: Coprevalent or correlated? Eur. J. Gastroenterol. Hepatol. 2018, 30, 979-985. [CrossRef]

11. Samji, N.S.; Verma, R.; Satapathy, S.K. Magnitude of nonalcoholic fatty liver disease: Western perspective. J. Clin. Exp. Hepatol. 2019, 9, 497-505. [CrossRef]

12. Milić, S.; Lulić, D.; Štimac, D. Non-alcoholic fatty liver disease and obesity: Biochemical, metabolic and clinical presentations. World J. Gastroenterol. 2014, 20, 9330-9337. [CrossRef] [PubMed]

13. Stephenson, K.; Kennedy, L.; Hargrove, L.; Demieville, J.; Thomson, J.; Alpini, G.; Francis, H. Updates on dietary models of non-alcoholic fatty liver disease: Current studies and insights. Gene Expr. J. Liver Res. 2018, 18, 5-17. [CrossRef] [PubMed]

14. Altinbas, A.; Sowa, J.P.; Hasenberg, T.; Canbay, A. The diagnosis and treatment of non-alcoholic fatty liver disease. Minerva Gastroenterol. Dietol. 2015, 61, 159-169. [PubMed]

15. Perdomo, C.M.; Frühbeck, G.; Escalada, J. Impact of nutritional changes on nonalcoholic fatty liver disease. Nutrients 2019, 11, 677. [CrossRef]

16. van der Windt, D.J.; Sud, V.; Zhang, H.; Tsung, A.; Huang, H. The effects of physical exercise on fatty liver disease. Gene Expr. 2018, 18, 89-101. [CrossRef] [PubMed]

17. Jeznach-Steinhagen, A.; Ostrowska, J.; Czerwonogrodzka-Senczyna, A.; Boniecka, I.; Shahnazaryan, U.; Kuryłowicz, A. Dietary and pharmacological treatment of nonalcoholic fatty liver disease. Medicina 2019, 55, 166. [CrossRef] [PubMed]

18. Sarwar, R.; Pierce, N.; Koppe, S. Obesity and nonalcoholic fatty liver disease: Current perspectives. Diabetes Metab. Syndr. Obes. Targets Ther. 2018, 11, 533-542. [CrossRef] [PubMed]

19. Xiao, J.; So, K.F.; Liong, E.C.; Tipoe, G.L. Recent Advances in the herbal treatment of Non-Alcoholic Fatty Liver Disease. J. Tradit. Complement. Med. 2013, 3, 88-94. [CrossRef] [PubMed]

20. Perumpail, B.J.; Li, A.A.; Iqbal, U.; Sallam, S.; Shah, N.D.; Kwong, W.; Cholankeril, G.; Kim, D.; Ahmed, A. Potential therapeutic benefits of herbs and supplements in patients with NAFLD. Diseases 2018, 6, 80. [CrossRef]

21. Huseini, H.F.; Larijani, B.; Heshmat, R.; Fakhrzadeh, H.; Radjabipour, B.; Toliat, T.; Raza, M. The efficacy of Silybum marianum (L.) Gaertn. (silymarin) in the treatment of type II diabetes: A randomized, double-blind, placebo-controlled, clinical trial. Phytother. Res. 2006, 20, 1036-1039. [CrossRef]

22. Theodotou, M.; Fokianos, K.; Moniatis, D.; Kadlenic, R.; Chrysikou, A.; Aristotelous, A.; Mouzouridou, A.; Diakides, J.; Stavrou, E. Effect of resveratrol on non-alcoholic fatty liver disease. Exp. Ther. Med. 2019, 18, 555-565. [CrossRef]

23. Godoy-Matos, A.F.; Silva Junior, W.S.; Valerio, C.M. NAFLD as a continuum: From obesity to metabolic syndrome and diabetes. Diabetol. Metab. Syndr. 2020, 12, 1-20. [CrossRef]

24. Dajani, A.; AbuHammour, A. Treatment of nonalcoholic fatty liver disease: Where do we stand? An overview. Saudi J. Gastroenterol. 2016, 22, 91-105. [CrossRef]

25. Petrásková, L.; Káňová, K.; Biedermann, D.; Křen, V.; Valentová, K. Simple and Rapid HPLC Separation and Quantification of Flavonoid, Flavonolignans, and 2,3-Dehydroflavonolignans in Silymarin. Foods 2020, 9, 116. [CrossRef]

26. Kazazis, C.E.; Evangelopoulos, A.A.; Kollas, A.; Vallianou, N.G. The therapeutic potential of milk thistle in diabetes. Rev. Diabet. Stud. RDS 2014, 11, 167-174. [CrossRef]

27. Ferenci, P. Silymarin in the treatment of liver diseases: What is the clinical evidence? Clin. Liver Dis. 2016, 7, 8-10. [CrossRef]

28. Pais, P.; D'Amato, M. In vivo efficacy study of milk thistle extract (ETHIS-094 ${ }^{\mathrm{TM}}$ ) in STAM ${ }^{\mathrm{TM}}$ model of nonalcoholic steatohepatitis. Drugs RED 2014, 14, 291-299. [CrossRef]

29. Gillessen, A.; Schmidt, H.H.J. Silymarin as supportive treatment in liver diseases: A narrative review. Adv. Ther. 2020, 37, 1279-1301. [CrossRef]

30. Cacciapuoti, F.; Scognamiglio, A.; Palumbo, R.; Forte, R.; Cacciapuoti, F. Silymarin in non alcoholic fatty liver disease. World J. Hepatol. 2013, 27, 109-113. [CrossRef] [PubMed]

31. Abenavoli, L.; Bellentani, S. Milk thistle to treat non-alcoholic fatty liver disease: Dream or reality? Expert Rev. Gastroenterol. Hepatol. 2013, 7, 677-679. [CrossRef]

32. Cicero, A.F.G.; Colletti, A.; Bellentani, S. Nutraceutical approach to non-alcoholic fatty liver disease (NAFLD): The available clinical evidence. Nutrients 2018, 10, 1153. [CrossRef] [PubMed]

33. Polyak, S.J.; Ferenci, P.; Pawlotsky, J.M. Hepatoprotective and antiviral functions of silymarin components in hepatitis C virus infection. Hepatology 2013, 57, 1262-1271. [CrossRef] 
34. Bhattacharya, S. Phytotherapeutic properties of milk thistle seeds: An overview. J. Adv. Pharm. Educ. 2011, 1, 69-79.

35. Frankenfield, D.C.; Muth, E.R.; Rowe, W.A. The Harris-Benedict studies of human basal metabolism: History and limitations. J. Am. Diet. Assoc. 1998, 98, 439-445. [CrossRef]

36. Marchesini, G.; Petta, S.; Dalle Grave, R. Diet, weight loss, and liver health in nonalcoholic fatty liver disease: Pathophysiology, evidence, and practice. Hepatology 2016, 63, 2032-2043. [CrossRef]

37. Mirmiran, P.; Amirhamidi, Z.; Ejtahed, H.S.; Bahadoran, Z.; Azizi, F. Relationship between Diet and Non-alcoholic Fatty Liver Disease: A Review Article. Iran. J. Public Health 2017, 46, 1007-1017.

38. Zelber-Sagi, S.; Ratziu, V.; Oren, R. Nutrition and physical activity in NAFLD: An overview of the epidemiological evidence. World J. Gastroenterol. 2011, 17, 3377-3389. [CrossRef] [PubMed]

39. Kenneally, S.; Sier, J.H.; Moore, J.B. Efficacy of dietary and physical activity intervention in non-alcoholic fatty liver disease: A systematic review. BMJ Open Gastroenterol. 2017, 4, e000139. [CrossRef]

40. World Health Organization (WHO). Body Mass Index-BMI. Available online: https://www.euro.who.int/en/health-topics/ disease-prevention/nutrition/a-healthy-lifestyle/body-mass-index-bmi (accessed on 10 February 2021).

41. de Koning, L.; Merchant, A.T.; Pogue, J.; Anand, S.S. Waist circumference and waist-to-hip ratio as predictors of cardiovascular events: Meta-regression analysis of prospective studies. Eur. Heart J. 2007, 28, 850-856. [CrossRef] [PubMed]

42. Głąbska, D.; Cackowska, K.; Guzek, D. Comparison of the body composition of Caucasian young normal body mass women, measured in the follicular phase, depending on the carbohydrate diet level. Medicina 2018, 54, 104. [CrossRef] [PubMed]

43. Kennedy, G.; Lee, W.T.K.; Termote, C.; Charrondière, R.; Tung, Y.E.A. Guidelines on Assessing Biodiverse Foods in Dietary Intake Surveys; Food and Agriculture Organization of the United Nations: Rome, Italy, 2017.

44. Kunachowicz, H.; Przygoda, B.; Nadolna, I.; Iwanow, K. Tabele Składu i Wartości Odżywczej Żywności; Wydawnictwo Lekarskie PZWL: Warsaw, Poland, 2017.

45. Jarosz, M. Normy Żywienia dla Populacji Polskiej; Instytut Żywności i Żywienia: Warsaw, Poland, 2017.

46. Keys, A.; Anderson, J.T.; Grande, F. Serum cholesterol response to changes in the diet: IV Particular saturated fatty acids in the diet. Metabolism 1965, 14, 776-787. [CrossRef]

47. Tahaei, S.; Sedighi, N.; Derogar, R.; Aslani, A.; Malekzadeh, R.; Merat, S. The effect of weight reduction on ultrasonographic findings of nonalcoholic fatty liver. Middle East J. Dig. Dis. 2010, 2, 5-8.

48. Hung, C.K.; Bodenheimer, H.C., Jr. Current treatment of nonalcoholic fatty liver disease/nonalcoholic steatohepatitis. Clin. Liver Dis. 2018, 22, 175-187. [CrossRef]

49. Dudekula, A.; Rachakonda, V.; Shaik, B.; Behari, J. Weight loss in nonalcoholic fatty liver disease patients in an ambulatory care setting is largely unsuccessful but correlates with frequency of clinic visits. PLoS ONE 2014, 9, e111808. [CrossRef]

50. Yoo, E.R.; Sallam, S.; Perumpail, B.J.; Iqbal, U.; Shah, N.D.; Kwong, W.; Cholankeril, G.; Kim, D.; Ahmed, A. When to initiate weight loss medications in the NAFLD population. Diseases 2018, 6, 91. [CrossRef]

51. Fogelholm, M.; Heinonen, M. Summer beef barbeque-A health risk? Duodecim Laaketieteellinen Aikakauskirja 2012, $128,1388-1391$.

52. Racette, S.B.; Weiss, E.P.; Schechtman, K.B.; Steger-May, K.; Villareal, D.T.; Obert, K.A.; Holloszy, J.O. Influence of weekend lifestyle patterns on body weight. Obesity 2008, 16, 1826-1830. [CrossRef]

53. Yanovski, J.A.; Yanovski, S.Z.; Sovik, K.N.; Nguyen, T.T.; O’Neil, P.M.; Sebring, N.G. A prospective study of holiday weight gain. N. Engl. J. Med. 2000, 342, 861-867. [CrossRef]

54. Turicchi, J.; O’Driscoll, R.; Horgan, G.; Duarte, C.; Palmeira, A.L.; Larsen, S.C.; Heitmann, B.L.; Stubbs, J. Weekly, seasonal and holiday body weight fluctuation patterns among individuals engaged in a European multi-centre behavioural weight loss maintenance intervention. PLoS ONE 2020, 15, e0232152. [CrossRef]

55. Díaz-Zavala, R.G.; Castro-Cantú, M.F.; Valencia, M.E.; Álvarez-Hernández, G.; Haby, M.M.; Esparza-Romero, J. Effect of the holiday season on weight gain: A narrative review. J. Obes. 2017, 2017, 2085136. [CrossRef]

56. van Wier, M.F.; Ariëns, G.A.M.; Dekkers, J.C.; Hendriksen, I.J.M.; Smid, T.; van Mechelen, W. Phone and e-mail counselling are effective for weight management in an overweight working population: A randomized controlled trial. BMC Public Health 2009, 9, 1-12. [CrossRef]

57. Bhutani, S.; Wells, N.; Finlayson, G.; Schoeller, D.A. Change in eating pattern as a contributor to energy intake and weight gain during the winter holiday period in obese adults. Int. J. Obes. 2020, 44, 1586-1595. [CrossRef]

58. Paul-Ebhohimhen, V.; Avenell, A. A systematic review of the effectiveness of group versus individual treatments for adult obesity. Obes. Facts 2009, 2, 17-24. [CrossRef] [PubMed]

59. Dalle Grave, R.; Sartirana, M.; Calugi, S. Personalized cognitive-behavioural therapy for obesity (CBT-OB): Theory, strategies and procedures. Biopsychosoc. Med. 2020, 14, 1-8. [CrossRef]

60. Perri, M.G.; Nezu, A.M.; Patti, E.T.; McCann, K.L. Effect of length of treatment on weight loss. J. Consult. Clin. Psychol. 1989, 57, 450-452. [CrossRef] [PubMed]

61. Renjilian, D.A.; Perri, M.G.; Nezu, A.M.; McKelvey, W.F.; Shermer, R.L.; Anton, S.D. Individual versus group therapy for obesity: Effects of matching participants to their treatment preferences. J. Consult. Clin. Psychol. 2001, 69, 717-721. [CrossRef]

62. Zhong, S.; Fan, Y.; Yan, Q.; Fan, X.; Wu, B.; Han, Y.; Zhang, Y.; Chen, Y.; Zhang, H.; Niu, J. The therapeutic effect of silymarin in the treatment of nonalcoholic fatty disease: A meta-analysis (PRISMA) of randomized control trials. Medicine 2017, 96 , e9061. [CrossRef] 
63. Meddeb, W.; Rezig, L.; Abderrabba, M.; Lizard, G.; Mejri, M. Tunisian milk thistle: An investigation of the chemical composition and the characterization of its cold-pressed seed oils. Int. J. Mol. Sci. 2017, 18, 2582. [CrossRef]

64. Amalan, V.; Vijayakumar, N.; Indumathi, D.; Ramakrishnan, A. Antidiabetic and antihyperlipidemic activity of p-coumaric acid in diabetic rats, role of pancreatic GLUT 2: In vivo approach. Biomed. Pharmacother. 2016, 84, 230-236. [CrossRef]

65. Cha, H.; Lee, S.; Lee, J.H.; Park, J.W. Protective effects of p-coumaric acid against acetaminophen-induced hepatotoxicity in mice. Food Chem. Toxicol. 2018, 121, 131-139. [CrossRef]

66. Liu, X.; Wang, D.; Zhang, D.; Lv, Y.; Wei, Y.; Wu, W.; Zhou, F.; Tang, M.; Mao, T.; Li, M.; et al. Inhibitory effect of blueberry polyphenolic compounds on oleic acid-induced hepatic steatosis in vitro. J. Agric. Food Chem. 2011, 59, 12254-12263. [CrossRef]

67. Famouri, F.; Salehi, M.M.; Rostampour, N.; Hashemi, E.; Shahsanaee, A. The effect of silymarin on non-alcoholic fatty liver disease of children. J. Herbmed. Pharmacol. 2017, 6, 16-20.

68. Hashemi, S.J.; Hajiani, E.; Sardabi, E.H. A placebo-controlled trial of Silymarin in patients with nonalcoholic fatty liver disease. Hepat. Mon. 2009, 9, 265-270.

69. Masoodi, M.; Rezadoost, A.; Panahian, M.; Vojdanian, M. Effects of Silymarin on reducing liver aminotransferases in patients with nonalcoholic fatty liver diseases. Govaresh 2013, 18, 181-185.

70. Taghvaei, T.; Bahar, A.; Hosseini, V.; Maleki, I.; Kasrai, M. Efficacy of Silymarin on treatment of nonalcoholic steatohepatitis. J. Mazandaran Univ. Med. Sci. 2013, 23, 164-171.

71. Memon, L.A.; Akbar, M.M.; Bhurgri, A.N. Effect of Silymarin therapy on liver aminotransferase in non-alcoholic fatty liver disease. Med Forum Mon. 2015, 26, 46-49.

72. Navarro, V.J.; Belle, S.H.; D'Amato, M.; Adfhal, N.; Brunt, E.M.; Fried, M.W.; Reddy, K.R.; Wahed, A.S.; Harrison, S. Silymarin in non-cirrhotics with non-alcoholic steatohepatitis: A randomized, double-blind, placebo controlled trial. PLoS ONE 2019, 14, e0221683. [CrossRef]

73. Kheong, C.W.; Mustapha, N.R.N.; Mahadeva, S. A randomized trial of Silymarin for the treatment of nonalcoholic steatohepatitis. Clin. Gastroenterol. Hepatol. 2017, 15, 1940-1949.e8. [CrossRef]

74. Kalopitas, G.; Antza, C.; Doundoulakis, I.; Siargkasb, A.; Kouroumalis, E.; Germanidis, G.; Samara, M.; Chourdakis, M. Impact of Silymarin in individuals with nonalcoholic fatty liver disease: A systematic review and meta-analysis. Nutrition 2021, 83, 111092. [CrossRef]

75. Anushiravani, A.; Haddadi, N.; Pourfarmanbar, M.; Mohammadkarimi, V. Treatment options for nonalcoholic fatty liver disease: A double-blinded randomized placebo-controlled trial. Eur. J. Gastroenterol. Hepatol. 2019, 31, 613-617. [CrossRef]

76. Riazi, K.; Raman, M.; Taylor, T.; Swain, M.G.; Shaheen, A.A. Dietary patterns and components in nonalcoholic fatty liver disease (NAFLD): What key messages can health care providers offer? Nutrients 2019, 11, 2878. [CrossRef]

77. Pickett-Blakely, O.; Young, K.; Carr, R.M. Micronutrients in nonalcoholic fatty liver disease pathogenesis. Cell. Mol. Gastroenterol. Hepatol. 2018, 6, 451-462. [CrossRef]

78. Da Silva, H.E.; Arendt, B.M.; Noureldin, S.A.; Therapondos, G.; Guindi, M.; Allard, J.P. A cross-sectional study assessing dietary intake and physical activity in Canadian patients with nonalcoholic fatty liver disease vs. healthy controls. J. Acad. Nutr. Diet. 2014, 114, 1181-1194. [CrossRef]

79. Emamat, H.; Farhadnejad, H.; Movahedian, M.; Tangestani, H.; Mirmiran, P.; Hekmatdoost, A. Dietary sodium intake in relation to non-alcoholic fatty liver disease risk: A case-control study. Nutr. Food Sci. 2020, 51, 541-550. [CrossRef]

80. Zhou, L.; Yang, Y.; Feng, Y.; Zhao, X.; Fan, Y.; Rong, J.; Zhao, L.; Yu, Y. Association between dietary sodium intake and non-alcoholic fatty liver disease in the US population. Public Health Nutr. 2020, 24, 993-1000. [CrossRef]

81. Morrissey, E.; Giltinan, M.; Kehoe, L.; Nugent, A.P.; McNulty, B.E.; Flynn, A.; Walton, J. Sodium and potassium intakes and their ratio in adults (18-90y): Findings from the Irish National Adult Nutrition Survey. Nutrients 2020, 12, 938. [CrossRef]

82. Chang, A.R.; Lazo, M.; Appel, L.J.; Gutierrez, O.M.; Grams, M.E. High dietary phosphorus intake is associated with all-cause mortality: Results from NHANES III. Am. J. Clin. Nutr. 2014, 99, 320-327. [CrossRef] [PubMed]

83. Li, W.; Zhu, X.; Song, Y.; Fan, L.; Wu, L.; Kabagambe, E.K.; Hou, L.; Shrubsole, M.J.; Liu, J.; Dai, Q. Intakes of magnesium, calcium and risk of fatty liver disease and prediabetes. Public Health Nutr. 2018, 21, 2088-2095. [CrossRef]

84. Wu, L.; Zhu, X.; Fan, L.; Kabagambe, E.K.; Song, Y.; Tao, M.; Zhong, X.; Hou, L.; Shrubsole, M.J.; Liu, J.; et al. Magnesium intake and mortality due to liver diseases: Results from the Third National Health and Nutrition Examination Survey Cohort. Sci. Rep. 2017, 7, 1-6. [CrossRef]

85. Himoto, T.; Masaki, T. Associations between zinc deficiency and metabolic abnormalities in patients with chronic liver disease. Nutrients 2018, 10, 88. [CrossRef] [PubMed]

86. Hariri, M.; Zohdi, S. Effect of vitamin D on non-alcoholic fatty liver disease: A systematic review of randomized controlled clinical trials. Int. J. Prev. Med. 2019, 10, 14. [CrossRef]

87. Shibata, K.; Sugita, C.; Sano, M.; Fukuwatari, T. Urinary excretion of B-group vitamins reflects the nutritional status of B-group vitamins in rats. J. Nutr. Sci. 2013, 2, e12. [CrossRef]

88. Yoo, E.R.; Kim, D.; Vazquez-Montesino, L.M.; Escober, J.A.; Li, A.A.; Tighe, S.P.; Fernandes, C.T.; Cholankeril, G.; Ahmed, A. Diet quality and its association with nonalcoholic fatty liver disease and all-cause and cause-specific mortality. Liver Int. 2020, 40, 815-824. [CrossRef] 
89. Park, S.Y.; Noureddin, M.; Boushey, C.; Wilkens, L.R.; Setiawan, V.W. Diet quality association with nonalcoholic fatty liver disease by cirrhosis status: The multiethnic cohort. Curr. Dev. Nutr. 2020, 4, nzaa024. [CrossRef]

90. McRae, M.R. Dietary Fiber Is Beneficial for the Prevention of Cardiovascular Disease: An Umbrella Review of Meta-analyses. J. Chiropr. Med. 2017, 16, 289-299. [CrossRef]

91. Obara-Gołębiowska, M.; Brycz, H.; Lipowska, M.; Lipowski, M. The role of motivation to reduce obesity among elderly people: Response to primingt in obese individuals. Int. J. Environ. Res. Public Health 2018, 15, 244. [CrossRef]

92. Rodriguez-Cristobal, J.J.; Alonso-Villaverde, C.; Panisello, J.M.; Travé-Mercade, P.; Rodriguez-Cortés, F.; Marsal, J.R.; Peña, E. Effectiveness of a motivational intervention on overweight/obese patients in the primary healthcare: A cluster randomized trial. BMC Fam. Pract. 2017, 18, 74. [CrossRef] [PubMed] 